\title{
A weighted eigencurve for Steklov problems with a potential
}

\author{
Liamidi Leadi and Aboubacar Marcos
}

\begin{abstract}
In this work, we study the existence of the principal eigencurves for a Steklov problem with an indefinite weight for homogeneous perturbation of the p-Laplacian operator. We then establish many properties of these eigencurves: continuity, differentiability and asymptotic behaviors. We also use our approach to get similar result when mixed DirichletSteklov boundary condition is considered.
\end{abstract}

Mathematics Subject Classification. 35J20, 35J70, 35P05, 35P30.

Keywords. Nonlinear Steklov eigenvalue problem, Dirichlet-Steklov boundary condition, $p$-Laplacian, Elliptic problem, Principal eigencurve, Indefinite weight.

\section{Introduction}

Let $\Omega \subset \mathbb{R}^{N}$ be a bounded smooth domain of class $C^{2, \alpha}(0<\alpha<1)$, with outward unit normal $\nu$ on the boundary $\partial \Omega ; 1<p<\infty$ and $\lambda, \mu$ are real parameters.

We investigate in the present work the following one parameter Steklov eigenvalue problem

$$
\begin{cases}\Delta_{p} u=V|u|^{p-2} u & \text { in } \Omega \\ |\nabla u|^{p-2} \frac{\partial u}{\partial \nu}=\lambda m(x)|u|^{p-2} u+\mu|u|^{p-2} u & \text { on } \partial \Omega\end{cases}
$$

where $\Delta_{p} u=\operatorname{div}\left(|\nabla u|^{p-2} \nabla u\right)$ is the $p$-Laplacian operator. The functions $V \in$ $L^{\infty}(\Omega)$ and $m \in C^{r}(\partial \Omega$ ) (for some $r \in(0,1)$ ) are allowed to change sign; $\mu$ stands for the eigenvalue while $\lambda$ is a real parameter.

We are interested in the weak solutions of (1.1), i.e. functions satisfying

$$
\begin{aligned}
& \int_{\Omega}|\nabla u|^{p-2} \nabla u \cdot \nabla v+\int_{\Omega} V(x)|u|^{p-2} u v \\
& \quad=\lambda \int_{\partial \Omega} m(x)|u|^{p-2} u v d \sigma+\mu \int_{\partial \Omega}|u|^{p-2} u v d \sigma \quad \forall v \in W^{1, p}(\Omega),
\end{aligned}
$$

where $d \sigma$ is the $(N-1)$ dimensional Hausdorff measure. 
This eigenvalue problem was first studied in [23] by M. W. Steklov in the particular case $p=2, V \equiv 0, \mu=0$ and $m \equiv 1$. Such problem is a modelization of an elastic membrane problem whose mass is concentrated on the boundary. For the nonlinear case, we refer to the work in [7] where the following problem has been considered

$$
\begin{cases}\Delta_{p} u=|u|^{p-2} u & \text { in } \Omega \\ |\nabla u|^{p-2} \frac{\partial u}{\partial \nu}=\lambda m(x)|u|^{p-2} u & \text { on } \partial \Omega .\end{cases}
$$

With the assumption $m^{+} \not \equiv 0$, it was proved in [7], by using Ljusternik-Schnirelman theory, the existence of a nondecreasing and unbounded sequence of positive eigenvalues. Moreover, the authors of [7] show that there exists a first positive eigenvalue which is simple, isolated and it is the only positive eigenvalue associated to an eigenfunction of constant sign. Similar results have been obtained later (when considered problem (1.1)) by [2] (when $V \equiv 0$ ), [11] (in the case $V \equiv 1$ ) and [27] (in the case $V \equiv 0$ and $\mu=0$ ). The case $V \geq 0$ is technically similar to the case $V \equiv 0$ since the energy function associated to the problem (1.1) defined by

$$
\Phi_{\lambda}(u)=\int_{\Omega}\left(|\nabla u|^{p}+V(x)|u|^{p}\right) d x-\lambda \int_{\partial \Omega} m(x)|u|^{p} d \sigma
$$

has similar properties with the energy function

$$
E(u)=\int_{\Omega}|\nabla u|^{p} d x-\lambda \int_{\partial \Omega} m(x)|u|^{p} d \sigma .
$$

So it will be very interesting to consider the case where the potential $V$ is an indefinite function.

We are interested in studying the principal eigenvalue of

$$
\begin{cases}\Delta_{p} u=V(x)|u|^{p-2} u & \text { in } \Omega \\ |\nabla u|^{p-2} \frac{\partial u}{\partial \nu}=\lambda m(x)|u|^{p-2} u & \text { on } \partial \Omega .\end{cases}
$$

For this study, we will apply the approach used by $[5,6,15,21]$ by considering the $\lambda$-depend eigenvalue problem (1.1). We denote by $\mu_{1}(\lambda)$ the first principal eigenvalue of (1.1) and letting varying the parameter $\lambda$, one gets the map $\lambda \mapsto \mu_{1}(\lambda)$ which is called, in the literature, the principal eigencurve of (1.1) and sets as

$$
\mu_{1}(\lambda)=\inf \left\{\Phi_{\lambda}(u): u \in W^{1, p}(\Omega) \text { and } \int_{\partial \Omega}|u|^{p} d \sigma=1\right\} .
$$

Knowing the properties and the behavior of this curve allows us to deduce the existence of zeros for the function $\mu_{1}(\lambda)$, which are principal eigenvalues of the problem (1.3). The results obtained here with a more general $V$ are new compared to those in $[2,7,11,27]$. Let notice that when $V$ and $m$ are indefinite, new difficulties related to the unboundedness of the functional $\Phi_{\lambda}$ arise (see for instance [14]).

The existence of the principal eigencurve $\mu_{1}(\lambda)$ of (1.1) is derived in Sect. 3. We also study there some of the properties of this eigenvalue as function of parameter $\lambda$. Using those properties of $\mu_{1}(\lambda)$, we derive a condition 
that guarantees the existence of principal eigenvalue for (1.3), which is in fact a zero for the function $\mu_{1}(\lambda)$. Section 4 is devoted to the search of the first nonprincipal eigenvalue of (1.3) by using a version of mountain pass theorem on $C^{1}$ manifolds along family of paths depending on the normalized eigenfunction (when it exists) or a free endpoints. Moreover, the technique used in Sect. 3 can be developed to get a related result for a mixed Dirichlet-Steklov eigenvalue problem in Sect. 5, i.e. the case where one can split the boundary of the domain up in two disjoint connected and closed sets. The Sect. 6 is an appendix which is devoted to some regularity result for a weak solution of (1.1). In the preliminary Sect. 2, we recall a useful result obtained in $[8,12]$, which will be used later.

Let us conclude this section with some general definitions relative to the Palais-Smale condition [in short the (PS) condition] and Palais-Smale-Cerami condition [abbreviated into (PSC) condition]. Let $E$ be a real Banach space and let $M:=\{u \in E: g(u)=1\}$ where $g \in C^{1}(E, \mathbb{R})$ and 1 is a regular value of $g$. Let $f \in C^{1}(E, \mathbb{R})$ and consider the restriction $\tilde{f}$ of $f$ to $M$. The differential $\tilde{f}^{\prime}$ at $u \in M$, has a norm which will be denoted by $\left\|\tilde{f}^{\prime}(u)\right\|_{\star}$ and which is given by the norm of the restriction of $f^{\prime}(u) \in E^{\star}$ to the tangent space of $M$ at $u$

$$
T u(M):=\left\{v \in E:\left\langle g^{\prime}(u), v\right\rangle=0\right\},
$$

where $\langle$,$\rangle denotes the pairing between E^{\star}$ and $E$.

We recall that $\tilde{f}$ is said to satisfy the $(P S)_{c}$ condition (resp. $(P S C)_{c}$ condition) at level $c \in \mathbb{R}$ if for any sequence $u_{k} \in M$ such that $\tilde{f}\left(u_{k}\right) \rightarrow c$ and $\left\|\tilde{f}^{\prime}\left(u_{k}\right)\right\|_{\star} \rightarrow 0$ (resp. $\tilde{f}\left(u_{k}\right) \rightarrow c$ and $\left(1+\left\|u_{k}\right\|_{E}\right)\left\|\tilde{f}^{\prime}\left(u_{k}\right)\right\|_{\star} \rightarrow 0$ ), one has that $u_{k}$ admits a convergent subsequence.

\section{Preliminaries}

Throughout this work, the functions $V$ and $m$ satisfy the following assumptions:

$\left(H_{V}\right) \quad V \in L^{\infty}(\Omega)$.

$\left(H_{m}\right) m \in C^{r}(\partial \Omega)$ (for some $\left.r \in(0,1)\right)$ and $m^{+} \not \equiv 0$ a.e in $\partial \Omega$.

Given $m \in C^{0, r}(\partial \Omega)$ we set

$$
\partial \Omega_{+} \stackrel{\text { def }}{=}\{x \in \partial \Omega: m(x)>0\}, \quad \partial \Omega_{-} \stackrel{\text { def }}{=}\{x \in \partial \Omega: m(x)<0\} .
$$

We start by recalling the following useful result in [12].

Theorem 2.1. [12] Let $\Omega \subset \mathbb{R}^{n}$ be a $C^{1, \alpha}$ domain, $0<\alpha<1$ and $V \in L^{\infty}(\Omega)$. Then the eigenvalue problem

$$
\begin{cases}-\Delta_{p} u+V(x)|u|^{p-2} u=\lambda|u|^{p-2} u & \text { in } \Omega \\ u=0 & \text { on } \partial \Omega\end{cases}
$$


has a unique eigenvalue $\lambda \equiv \lambda_{1}^{D}(V)$ which is defined by

$$
\lambda_{1}^{D}(V) \stackrel{\text { def }}{=} \inf \left\{\int_{\Omega}\left(|\nabla u|^{p}+V(x)|u|^{p}\right) d x ; u \in W_{0}^{1, p}(\Omega) \text { and } \int_{\Omega}|u|^{p} d x=1\right\}
$$

with the property of exhibiting a positive eigenfunction $\phi \in W_{0}^{1, p}(\Omega)$. Moreover $\lambda_{1}^{D}(V)$ is simple and isolated. The positive eigenfunctions satisfy $\phi \in C^{1, \beta}(\bar{\Omega})$ for some $0<\beta<1$ and $\frac{\partial \phi}{\partial \mu}<0$ on $\partial \Omega$.

\section{Existence of principal eigenvalues}

Our purpose in this section is to derive an existence result concerning principal eigenvalues for (3.14) (see below) by using the approach in $[5,6,8,21]$ which consisting of obtaining principal eigenvalues as zeros of the eigencurve. We assume that $V$ and $m$ satisfy $\left(H_{V}\right)$ and $\left(H_{m}\right)$ respectively and consider first the problem (1.1).

We recall that, for a fixed real $\lambda, \mu=\mu(\lambda)$ is an eigenvalue of (1.1) if and only if there exists $u \in W^{1, p}(\Omega), u \neq \equiv 0$ such that

$$
\int_{\Omega}|\nabla u|^{p-2} \nabla u \nabla v+\int_{\Omega} V|u|^{p-2} u v=\int_{\partial \Omega}(\lambda m(x)+\mu)|u|^{p-2} u v .
$$

for all $v \in W^{1, p}(\Omega) ; u$ is then called an eigenfunction associated to $\mu$. We will use a variational approach to study (1.1). For that purpose, we define the following functional

$$
\Phi_{\lambda}(u) \stackrel{\text { def }}{=} \int_{\Omega}\left(|\nabla u|^{p}+V(x)|u|^{p}\right) d x-\lambda \int_{\partial \Omega} m(x)|u|^{p} d \sigma
$$

which is $C^{1}$ and well defined on $W^{1, p}(\Omega)$. It follows from standard arguments (Lagrange multiplier rule) that the eigenvalues $\mu$ of (1.1) correspond to critical value of $\tilde{\Phi}_{\lambda}$, where $\tilde{\Phi}_{\lambda}$ denotes the restriction of $\Phi_{\lambda}$ to the manifold

$$
\mathcal{S} \stackrel{\text { def }}{=}\left\{u \in W^{1, p}(\Omega): \int_{\partial \Omega}|u|^{p} d \sigma=1\right\} .
$$

Remark 3.1. Using the results of Theorem 6.1, (see the appendix in Section 6) and Theorem 2 in [19], we conclude that such a weak solution $u$ of (1.1) belongs to $C^{1, \alpha}(\bar{\Omega})$ for some $0<\alpha<1$.

The following theorem is the main result in this section. We then generalize the result of Theorem 6 of [13] where the linear case is considered.

Theorem 3.1. Let $\Omega$ be a bounded smooth domain of class $C^{2, \alpha}(0<\alpha<1)$ in $\mathbb{R}^{N}$ with boundary $\partial \Omega$. Assume that the weights $V$ and $m$ satisfy $\left(H_{V}\right)$ and $\left(H_{m}\right)$, respectively. Then for every $\lambda \in \mathbb{R},(1.1)$ admits a principal eigenvalue $\mu_{1}(\lambda)$, i.e. an eigenvalue associated to a one-signed eigenfunction if and only if

$$
\lambda_{1}^{D}(V)>0 .
$$

Moreover $\mu_{1}(\lambda)$ is simple and there is no other principal eigenvalue of (1.1). 
Proof. For a fixed $\lambda$, the existence of a principal eigenvalue $\mu_{1}(\lambda)$ for (1.1), with associated positive eigenfunction $u_{\lambda}$ provides a positive strict supersolution to the equation

$$
-\Delta_{p} u+V(x)|u|^{p-2} u=0
$$

in $\Omega$ since $u_{\lambda}$ must also be positive on $\partial \Omega$. Indeed since $u_{\lambda}$ is nonnegative in $\Omega$, by the maximum principle of Vazquez [28], we deduce that $u_{\lambda}>0$ a.e in $\Omega$.We will prove that $u_{\lambda}$ is postiche up to the boundary. Let $\varepsilon>0$ and set $u_{\varepsilon}=\frac{u_{\lambda}}{u_{\lambda}+\varepsilon}$. Then using the dominated convergence theorem we have $u_{\varepsilon} \rightarrow 1_{\Omega}$ in $L^{p}(\Omega)$ and next $\nabla u_{\varepsilon} \rightarrow 0$, a.e in $\Omega$ as $\varepsilon \rightarrow 0$. Taking $v=\frac{1}{\left(u_{\lambda}+\varepsilon\right)^{p-1}}$ as test function in (3.1) (where we take $u=u_{\lambda}$ and $\mu=\mu_{1}(\lambda)$ ) we have

$$
\begin{gathered}
(p-1) \int_{\Omega} \frac{\left|\nabla u_{\lambda}\right|^{p}}{\left(u_{\lambda}+\varepsilon\right)^{p}} d x=\int_{\Omega} V\left(\frac{u_{\lambda}}{u_{\lambda}+\varepsilon}\right)^{p-1} d x \\
-\int_{\partial \Omega}\left[\lambda m+\mu_{1}(\lambda)\right]\left(\frac{u_{\lambda}}{u_{\lambda}+\varepsilon}\right)^{p-1} d \sigma .
\end{gathered}
$$

Using Fatou's lemma we deduce that

$$
\begin{gathered}
(p-1) \int_{\Omega} \frac{\left|\nabla u_{\lambda}\right|^{p}}{u_{\lambda}^{p}} d x \leq \lim _{\varepsilon \rightarrow 0}\left[\int_{\Omega} V\left(\frac{u_{\lambda}}{u_{\lambda}+\varepsilon}\right)^{p-1} d x\right. \\
\left.-\int_{\partial \Omega}\left[\lambda m+\mu_{1}(\lambda)\right]\left(\frac{u_{\lambda}}{u_{\lambda}+\varepsilon}\right)^{p-1} d \sigma\right] \in \mathbb{R} .
\end{gathered}
$$

Hence

$$
\left|\nabla u_{\varepsilon}\right|^{p}=\left(\frac{\varepsilon}{u_{\lambda}+\varepsilon}\right)^{p} \frac{\left|\nabla u_{\lambda}\right|^{p}}{\left(u_{\lambda}+\varepsilon\right)^{p}} \leq \frac{\left|\nabla u_{\lambda}\right|^{p}}{u_{\lambda}^{p}} \in L^{1}(\Omega) .
$$

Again by the dominated convergence theorem, we deduce that $u_{\varepsilon} \rightarrow 1_{\Omega}$ in $W^{1, p}(\Omega)$ as $\varepsilon \rightarrow 0$. Finally, using the continuity of the trace mapping we have that $u_{\varepsilon} \rightarrow 1_{\partial \Omega}$ in $L^{1}(\partial \Omega)$ as $\varepsilon \rightarrow 0$, which allows us to conclude that $u_{\lambda}>0$ on $\partial \Omega$, so $u_{\lambda}>0$ in $\bar{\Omega}$. Using Theorem 2 of [12], we deduce that $\lambda_{1}^{D}(V)>0$.

We will now show that condition (3.3) is sufficient to have coercivity of the functional $\Phi_{\lambda}$, and since it is sequentially weakly lower semicontinuous in $W^{1, p}(\Omega)$, this yields the existence of a principal eigenvalue for problem (1.1) (cf. [24]).

Let show that $\Phi_{\lambda}$ is coercive on $\mathcal{S}$. Indeed, suppose that there exists a sequence $u_{n} \in \mathcal{S}$ such that

$$
\begin{aligned}
& \left\|u_{n}\right\|_{W^{1, p}(\Omega)}^{p}=\int_{\Omega}\left(\left|\nabla u_{n}\right|^{p}+\left|u_{n}\right|^{p}\right) d x \rightarrow+\infty \text { and } \\
& \Phi_{\lambda}\left(u_{n}\right)=\int_{\Omega}\left(\left|\nabla u_{n}\right|^{p}+V(x)\left|u_{n}\right|^{p}\right)-\lambda \int_{\partial \Omega} m(x)\left|u_{n}\right|^{p} d \sigma \leq K
\end{aligned}
$$

for some constant $K$. Since $V$ is bounded, this implies that $t_{n}=\left(\int_{\Omega}\left|u_{n}\right|^{p}\right)^{\frac{1}{p}} \rightarrow$ $+\infty$. Indeed assume that $t_{n}$ is bounded. Then $\int_{\Omega} V(x)\left|u_{n}\right|^{p}$ remains bounded, 
and since $u_{n} \in \mathcal{S}$, we deduce that

$$
\int_{\Omega}\left|\nabla u_{n}\right|^{p}=\Phi_{\lambda}\left(u_{n}\right)-\int_{\Omega} V(x)\left|u_{n}\right|^{p}+\lambda \int_{\partial \Omega} m(x)\left|u_{n}\right|^{p} d \sigma
$$

is bounded, a contradiction with the assumption $\left\|u_{n}\right\|_{W^{1, p}(\Omega)} \rightarrow+\infty$.

Setting $v_{n}=\frac{u_{n}}{t_{n}}$, we deduce that $\int_{\Omega}\left|v_{n}\right|^{p} d x=1$ and

$$
\left.\left|\int_{\partial \Omega} m(x)\right| v_{n}\right|^{p} d \sigma \mid \leq \frac{\|m\|_{\infty, \partial \Omega}}{t_{n}^{p}} \rightarrow 0, \quad \Phi_{\lambda}\left(v_{n}\right)=\frac{\Phi_{\lambda}\left(u_{n}\right)}{t_{n}^{p}} \leq \frac{K}{t_{n}^{p}} \rightarrow 0,
$$

where $\|\cdot\|_{\infty, \partial \Omega}$ denotes the $L^{\infty}(\partial \Omega)$-norm. Consequently $\int_{\Omega}\left|\nabla v_{n}\right|^{p} d x$ remains bounded and up to a subsequence $v_{n}$ converges to some $v$ weakly in $W^{1, p}(\Omega)$ and strongly in $L^{p}(\Omega)$ and in $L^{p}(\partial \Omega)$. Thus $\int_{\Omega}|v|^{p} d x=1$ and $\int_{\partial \Omega}|v|^{p} d \sigma=0$ (here we use the fact that $u_{n} \in \mathcal{S}$ ), which implies that $v \neq 0$ in $\Omega$ and $v \equiv 0$ in $\partial \Omega$, thus $v \in W_{0}^{1, p}(\Omega)$.

Moreover, from the boundednes of $\Phi_{\lambda}\left(u_{n}\right)$, we deduce that

$$
\lambda_{1}^{D}(V)=\lambda_{1}^{D}(V) \int_{\Omega}|v|^{p} d x \leq \int_{\Omega}\left(|\nabla v|^{p}+V|v|^{p}\right) d x \leq \liminf \Phi_{\lambda}\left(v_{n}\right) \leq 0,
$$

a contradiction since $v \neq 0$ in $\Omega$ and $\lambda_{1}^{D}(V)>0$.

On the other hand, since $\Phi_{\lambda}$ is sequentially weakly lower semicontinuous, $\Phi_{\lambda}$ attains a minimum value $\mu_{1}(\lambda)$ in $\mathcal{S}$, with

$$
\mu_{1}(\lambda) \stackrel{\text { def }}{=} \inf _{u \in \mathcal{S}} \Phi_{\lambda}(u) .
$$

By Lagrange multipliers rule, $\mu_{1}(\lambda)$ defined in (3.4) is an eigenvalue of (1.1). Since $\Phi_{\lambda}(|u|)=\Phi_{\lambda}(u)$, we can suppose that the associated eigenfunction is nonnegative. Using the Harnack inequality as in [22, Theorems 5,6 and 9, pp 264-270] and the first part of the proof, we conclude that the eigenfunction is positive up to the boundary and then the eigenvalue $\mu_{1}(\lambda)$ is principal.

We will use Picone's identity (see[1]) to prove simplicity of $\mu_{1}(\lambda)$ and non existence of further principal eigenvalues.

Indeed let $L$ and $R$ be defined, for any $\xi \geq 0$ and $\phi>0$ two a.e. differentiable functions, as

$$
\begin{aligned}
& L(\xi, \phi):=|\nabla \xi|^{p}+(p-1) \frac{\xi^{p}}{\phi^{p}}|\nabla \phi|^{p}-p \frac{\xi^{p-1}}{\phi^{p-1}}|\nabla \phi|^{p-2} \nabla \phi \nabla \xi \\
& R(\xi, \phi):=|\nabla \xi|^{p}-|\nabla \phi|^{p-2} \nabla\left(\frac{\xi^{p}}{\phi^{p-1}}\right) \nabla \phi .
\end{aligned}
$$

Picone's Identity (cf. [1]) claims that $0 \leq L(\xi, \phi)=R(\xi, \phi)$ and $L(\xi, \phi)=0$ if and only if $\xi$ and $\phi$ are colinear.

For a fixed $\lambda$, assume that $u, v \in W^{1, p}(\Omega)$ are two eigenfunctions of (1.1) associated respectively to $\mu_{1}(\lambda)$ and $\beta$. Assume also that $u \geq 0$ and $v>0$ in $\Omega$. From the definition of $\mu_{1}(\lambda)$ (cf.(3.4)), we derive that $\beta \geq \mu_{1}(\lambda)$. We then 
apply Picone's Identity to $u$ and $v+\varepsilon$ for any $\epsilon>0$, and obtain

$$
\begin{aligned}
0 \leq & \int_{\Omega} L(u, v+\varepsilon) d x=\int_{\Omega} R(u, v+\varepsilon) d x \\
= & \int_{\Omega}\left[|\nabla u|^{p}-|\nabla v|^{p-2} \nabla v \cdot \nabla\left(\frac{u^{p}}{(v+\varepsilon)^{p-1}}\right)\right] d x \\
= & \lambda \int_{\partial \Omega} m(x)|u|^{p} d \sigma+\mu_{1}(\lambda) \int_{\partial \Omega}|u|^{p} d \sigma-\int_{\Omega} V|u|^{p} d x \\
& -\lambda \int_{\partial \Omega} m(x) v^{p-1} \frac{u^{p}}{(v+\varepsilon)^{p-1}} d \sigma-\beta \int_{\partial \Omega} v^{p-1} \frac{u^{p}}{(v+\varepsilon)^{p-1}} d \sigma \\
& +\int_{\Omega} V v^{p-1} \frac{u^{p}}{(v+\varepsilon)^{p-1}}
\end{aligned}
$$

Passing to the limit, as $\varepsilon \rightarrow 0$, yields

$$
0 \leq \int_{\Omega} L(u, v) d x \leq\left(\mu_{1}(\lambda)-\beta\right) \int_{\partial \Omega}|u|^{p} d x .
$$

Consequently, if $\beta=\mu_{1}(\lambda)$ then $L(u, v)=0$ and hence $u=c v$ for some positive constant $c$.

From now we will denote by $\varphi_{\lambda}$ the eigenfunction associated to $\mu_{1}(\lambda)$ with $\int_{\partial \Omega} \varphi_{\lambda} d \sigma=1$ and $\varphi_{\lambda}>0$ on $\bar{\Omega}$. In the following proposition we give some properties of the function $\lambda \mapsto \mu_{1}(\lambda)$.

Proposition 3.2. (1) The function $\lambda \mapsto \mu_{1}(\lambda)$ is concave and differentiable.

Moreover

$$
\mu_{1}^{\prime}(\lambda)=-\int_{\partial \Omega} m(x) \varphi_{\lambda}^{p}(x) d \sigma \text { for all } \lambda \in \mathbb{R} .
$$

(2) If $m^{+} \not \equiv 0$ (resp. $m^{-} \not \equiv 0$ ) then $\lim _{\lambda \rightarrow+\infty} \mu_{1}(\lambda)=-\infty$ (resp. $\left.\lim _{\lambda \rightarrow-\infty} \mu_{1}(\lambda)=-\infty\right)$.

(3) If $m^{-} \equiv 0$ then $\mu_{1}$ is strictly decreasing.

(4) $\sup _{\lambda \in \mathbb{R}} \mu_{1}(\lambda)=\beta(V, m)$ where

$$
\begin{aligned}
& \beta(V, m)=\infty \quad \text { if } m(x)>0 \quad \text { a.e. in } \partial \Omega \quad \text { or } \\
& \beta(V, m) \stackrel{\text { def }}{=} \inf _{u \in \mathcal{A}} E_{V}(u) \quad \text { if } 0<\left|\partial \Omega_{+}\right|<|\partial \Omega|,
\end{aligned}
$$

with $E_{V}(u)=\int_{\Omega}\left(|\nabla u|^{p}+V|u|^{p}\right) d x$ and

$$
\mathcal{A}=\left\{u \in W^{1, p}(\Omega), \quad \int_{\partial \Omega}|u|^{p} d \sigma=1 \text { and } \int_{\partial \Omega} m|u|^{p} d \sigma=0\right\} .
$$

Proof. The proof is partly adapted from an analogous result in [8].

(1) The concavity of $\mu_{1}$ follows the fact that $\mu_{1}(\lambda)$ is the infimum of a family of affine functions, and consequently $\mu_{1}$ is continuous on $\mathbb{R}$. Now let $\lambda_{n} \rightarrow \lambda$ and denote by $\varphi_{n}, \varphi$ the $L^{p}(\partial \Omega)$-normalized positive eigenfunction associated to $\lambda_{n}, \lambda$ respectively. Let $\Lambda \subset \mathbb{R}$ be bounded and let $\lambda_{n} \in \Lambda$. Since 


$$
\mu_{1}\left(\lambda_{n}\right)=\int_{\Omega}\left(\left|\nabla \varphi_{n}\right|^{p}+V\left|\varphi_{n}\right|^{p}\right) d x-\lambda_{n} \int_{\partial \Omega} m(x)\left|\varphi_{n}\right|^{p} d \sigma \leq K \quad \text { (constant) }
$$

(by the continuity of $\mu_{1}$ ), one deduces that $\varphi_{n}$ is bounded in $W^{1, p}(\Omega)$ (here we use the fact that $V \in L^{\infty}(\Omega), m \in L^{\infty}(\partial \Omega)$ and $\left.\varphi_{n} \in \mathcal{S}\right)$. Thus for some $\tilde{\varphi} \in W^{1, p}(\Omega), \varphi_{n}$ converges to $\tilde{\varphi}$ weakly in $W^{1, p}(\Omega)$ and strongly in $L^{p}(\Omega)$ and in $L^{p}(\partial \Omega)$ as $\lambda_{n} \rightarrow \lambda \in \bar{\Lambda}$. Next, taking $\varphi_{k}-\varphi_{l}$ as a test function for $\varphi_{k}$ in (1.1), one has

$$
\begin{aligned}
& \int_{\Omega}\left|\nabla \varphi_{k}\right|^{p-2} \nabla \varphi_{k} \cdot \nabla\left(\varphi_{k}-\varphi_{l}\right)+\int_{\Omega} V(x) \varphi_{k}^{p-1}\left(\varphi_{k}-\varphi_{l}\right) \\
& \quad=\lambda_{k} \int_{\partial \Omega} m(x) \varphi_{k}^{p-1}\left(\varphi_{k}-\varphi_{l}\right) d \sigma+\mu_{1}\left(\lambda_{k}\right) \int_{\partial \Omega} \varphi_{k}^{p-1}\left(\varphi_{k}-\varphi_{l}\right) d \sigma .
\end{aligned}
$$

Then, from the monotonicity of the function $x \mapsto\|x\|^{p-2} x$, we have

$$
\begin{aligned}
0 \leq J\left(\varphi_{k}, \varphi_{l}\right) \stackrel{\text { def }}{=} & \int_{\Omega}\left(\left|\nabla \varphi_{k}\right|^{p-2} \nabla \varphi_{k}-\left|\nabla \varphi_{l}\right|^{p-2} \nabla \varphi_{l}\right) \cdot \nabla\left(\varphi_{k}-\varphi_{l}\right) d x \\
= & -\int_{\Omega} V(x)\left(\varphi_{k}^{p-1}-\varphi_{l}^{p-1}\right)\left(\varphi_{k}-\varphi_{l}\right) d x \\
& +\lambda_{k} \int_{\partial \Omega} m(x)\left(\varphi_{k}^{p-1}-\varphi_{l}^{p-1}\right)\left(\varphi_{k}-\varphi_{l}\right) d \sigma \\
& +\left(\lambda_{k}-\lambda_{l}\right) \int_{\partial \Omega} m(x) \varphi_{l}^{p-1}\left(\varphi_{k}-\varphi_{l}\right) d \sigma+\mu_{1}\left(\lambda_{k}\right) \\
& \times \int_{\partial \Omega}\left(\varphi_{k}^{p-1}-\varphi_{l}^{p-1}\right)\left(\varphi_{k}-\varphi_{l}\right) d \sigma \\
& +\left[\mu_{1}\left(\lambda_{k}\right)-\mu_{1}\left(\lambda_{l}\right)\right] \int_{\Omega} \varphi_{l}^{p-1}\left(\varphi_{k}-\varphi_{l}\right) d \sigma
\end{aligned}
$$

and next, we get

$$
\begin{aligned}
0 \leq & J\left(\lambda_{k}, \lambda_{l}\right) \\
\leq & \int_{\Omega}|V(x)|\left|\varphi_{k}^{p-1}-\varphi_{l}^{p-1}\right|\left|\varphi_{k}-\varphi_{l}\right| d x+\left|\lambda_{k}-\lambda_{l}\right| \int_{\partial \Omega}|m(x)| \varphi_{l}^{p-1}\left|\varphi_{k}-\varphi_{l}\right| d \sigma \\
& +\left|\lambda_{k}\right| \int_{\partial \Omega}|m(x)|\left|\varphi_{k}^{p-1}-\varphi_{l}^{p-1}\right|\left|\varphi_{k}-\varphi_{l}\right| d \sigma+\left|\mu_{1}\left(\lambda_{k}\right)\right| \int_{\partial \Omega}\left|\varphi_{k}^{p-1}-\varphi_{l}^{p-1}\right| \mid \\
& \times \varphi_{k}-\varphi_{l}|d \sigma+| \mu_{1}\left(\lambda_{k}\right)-\mu_{1}\left(\lambda_{l}\right)\left|\int_{\partial \Omega} \varphi_{l}^{p-1}\right| \varphi_{k}-\varphi_{l} \mid d \sigma
\end{aligned}
$$

Using Hölder inequality, the hypothesis $\left(H_{V}\right),\left(H_{m}\right)$ and the fact that $\varphi_{k}, \varphi_{l} \in \mathcal{S}$, one derives that the right hand side of (3.9) converges to 0 , so that $J\left(\varphi_{k}, \varphi_{l}\right) \rightarrow 0$ as $k, l \rightarrow+\infty$.

To go on with the proof, we observe that for some constant $c=c(p)$ and for any $a, b \in \mathbb{R}^{N}$,

$$
\|a-b\|^{p} \leq c\left\{\left(\|a\|^{p-2} a-\|b\|^{p-2} b\right)(a-b)\right\}^{s / 2}\left(\|a\|^{p}+\|b\|^{p}\right)^{1-s / 2},
$$

where $s=p$ if $p \in] 1,2[$ and $s=2$ if $p \geq 2$ (cf. [16,17]). Applying (3.10) and Hölder inequality, one easily derives that $\nabla \varphi_{n} \rightarrow \nabla \tilde{\varphi}$ in $L^{p}(\Omega)$ and 
consequently $\varphi_{n} \rightarrow \tilde{\varphi}$ in $W^{1, p}(\Omega)$. On the other hand, one has

$$
\begin{aligned}
\mu_{1}(\lambda) & =\lim _{n \rightarrow+\infty} \mu_{1}\left(\lambda_{n}\right) \\
& =\lim _{n \rightarrow+\infty} \int_{\Omega}\left[\left|\nabla \varphi_{n}\right|^{p}+V(x) \varphi_{n}^{p}\right]-\lambda_{n} \int_{\partial \Omega} m(x) \varphi_{n}^{p} d \sigma \\
& =\Phi_{\lambda}(\tilde{\varphi})
\end{aligned}
$$

and since $\int_{\partial \Omega} \tilde{\varphi}^{p} d \sigma=1$, one derives from Proposition 3.1 that $\tilde{\varphi}=\varphi_{\lambda}$.

To prove the differentiability of $\mu_{1}(\lambda)$, let write

$$
\begin{aligned}
\mu_{1}\left(\lambda_{n}\right) & =\int_{\Omega}\left[\left|\nabla \varphi_{n}\right|^{p}+V(x) \varphi_{n}^{p}\right] d x-\lambda_{n} \int_{\partial \Omega} m(x) \varphi_{n}^{p} d \sigma \\
& =\Phi_{\lambda}\left(\varphi_{n}\right)-\left(\lambda_{n}-\lambda\right) \int_{\partial \Omega} m(x) \varphi_{n}^{p} d \sigma \\
& \geq \mu_{1}(\lambda)-\left(\lambda_{n}-\lambda\right) \int_{\partial \Omega} m(x) \varphi_{n}^{p} d \sigma .
\end{aligned}
$$

Interchanging the role of $\lambda$ and $\lambda_{n}$, and replacing $\varphi_{n}$ by $\varphi_{\lambda}$ in the last inequality we have

$$
-\left(\lambda_{n}-\lambda\right) \int_{\partial \Omega} m(x) \varphi_{n}^{p} d \sigma \leq \mu_{1}\left(\lambda_{n}\right)-\mu_{1}(\lambda) \leq-\left(\lambda_{n}-\lambda\right) \int_{\partial \Omega} m(x) \varphi_{\lambda}^{p} d \sigma .
$$

Dividing by $\left(\lambda_{n}-\lambda\right)$ an letting $\lambda_{n} \rightarrow \lambda$, we obtain (3.5).

(2) Since $m^{+} \not \equiv 0$, there exists a function $u_{0} \in W^{1, p}(\Omega)$ such that $\int_{\partial \Omega} m\left|u_{0}\right|^{p} d \sigma>0$ and $\int_{\partial \Omega}\left|u_{0}\right|^{p} d \sigma=1$, and consequently for all $\lambda>0$,

$$
\mu_{1}(\lambda) \leq \Phi_{\lambda}\left(u_{0}\right) \longrightarrow-\infty \text { as } \lambda \rightarrow+\infty .
$$

A similar proof holds if $m^{-} \not \equiv 0$.

(3) This result is clear from (3.5) since $\varphi_{\lambda}>0$ on $\bar{\Omega}$ and $m^{+} \not \equiv 0$ on $\partial \Omega$.

(4) Let us proof that $\sup _{\lambda \in \mathbb{R}} \mu_{1}(\lambda)=\beta(V, m)$.

(a) If $m \geq 0$ on $\partial \Omega$, we know that $\mu_{1}$ is strictly decreasing on $\mathbb{R}$ and consequently we get

$$
\sup _{\lambda \in \mathbb{R}} \mu_{1}(\lambda)=\lim _{\lambda \rightarrow-\infty} \mu_{1}(\lambda) .
$$

Let $\lambda_{n} \rightarrow-\infty$, as $n \rightarrow+\infty$ and denote by $\varphi_{n}$ the associated eigenfunction with $\int_{\partial \Omega}\left|\varphi_{n}\right|^{p} d \sigma=1$.

(i) If $m>0$ on $\partial \Omega$, we know by definition that $\beta(V, m)=\infty$. Assume by contradiction that $\sup \mu_{1}(\lambda)<\infty$. Consequently we have

$$
\mu_{1}\left(\lambda_{n}\right)=E_{V}\left(\varphi_{n}\right)-\lambda_{n} \int_{\partial \Omega} m\left|\varphi_{n}\right|^{p} d \sigma \leq K(\text { for some constant } K)
$$

we claim that $t_{n}=\left(\int_{\Omega}\left|\varphi_{n}\right|^{p} d x\right)^{1 / p} \rightarrow+\infty$. Let prove this assertion. If $t_{n}$ is bounded then $\int_{\Omega} V\left|\varphi_{n}\right|^{p} d x$ is bounded and since we have 
$\int_{\Omega}\left|\nabla \varphi_{n}\right|^{p} d x \leq \mu_{1}\left(\lambda_{n}\right)-\int_{\Omega} V\left|\varphi_{n}\right|^{p} d x, \quad$ for all $\lambda_{n} \leq 0$

then we deduce that $\int_{\Omega}\left|\nabla \varphi_{n}\right|^{p} d x$ is bounded. Thus $\varphi_{n}$ is bounded in $W^{1, p}(\Omega)$ and consequently $\varphi_{n}$ converges to $\varphi_{0}$ weakly in $W^{1, p}(\Omega)$ and strongly in $L^{p}(\Omega)$ and in $L^{p}(\partial \Omega)$. We deduce that $\int_{\partial \Omega}\left|\varphi_{0}\right|^{p} d \sigma=1$, so $\varphi_{0} \not \equiv 0$ in $\partial \Omega$.

Moreover we have $\int_{\partial \Omega} m\left|\varphi_{n}\right|^{p} d \sigma \rightarrow \int_{\partial \Omega} m\left|\varphi_{0}\right|^{p} d \sigma>0$ and

$\lim _{n \rightarrow+\infty} \mu_{1}\left(\lambda_{n}\right)=\lim \left[E_{V}\left(\varphi_{n}\right)-\lambda_{n} \int_{\partial \Omega} m\left|\varphi_{n}\right|^{p} d \sigma\right]=+\infty$,

a contradiction. So $t_{n} \rightarrow \infty$, as $n \rightarrow \infty$ and then defining $v_{n}=\frac{\varphi_{n}}{t_{n}}$, we have $\int_{\Omega}\left|v_{n}\right|^{p} d x=1$ and $\int_{\Omega} V\left|v_{n}\right|^{p} d x$ bounded. Moreover, for all $\lambda_{n} \leq 0$

$$
E_{V}\left(v_{n}\right) \leq \Phi_{\lambda_{n}}\left(v_{n}\right)=\frac{\mu_{1}\left(\lambda_{n}\right)}{t_{n}^{p}} \leq \frac{K}{t_{n}^{p}} \rightarrow 0,
$$

we deduce that $v_{n}$ is bounded in $W^{1, p}(\Omega)$. Consequently $v_{n}$ converges to some $v_{0}$, weakly in $W^{1, p}(\Omega)$ and strongly in $L^{p}(\Omega)$ and in $L^{p}(\partial \Omega)$. This implies that $v_{0} \not \equiv 0$ in $\Omega$ since $\int_{\Omega}\left|v_{0}\right|^{p} d x=1$.

On the other hand, since $\int_{\partial \Omega}\left|v_{n}\right|^{p} d \sigma=\frac{1}{t_{n}^{p}} \rightarrow 0$, we deduce that $v_{0} \equiv 0$ on $\partial \Omega$ and consequently $v_{0} \in W_{0}^{1, p}(\Omega)$.

Finally we have

$$
\lambda_{1}^{D}(V)=\lambda_{1}^{D}(V) \int_{\Omega}\left|v_{0}\right|^{p} d x \leq E_{V}\left(v_{0}\right) \leq \liminf E_{V}\left(v_{n}\right) \leq 0,
$$

a contradiction.

(ii) If $m \geq 0$ on $\partial \Omega$ we set $\partial \Omega_{0}=\partial \Omega \backslash \operatorname{Supp}(m)$. Since $\left|\partial \Omega^{+}\right|<|\partial \Omega|$ we have $\left|\partial \Omega_{0}\right|>0$ and then we can construct $u_{0} \in W^{1, p}(\Omega)$ such that $u_{0} \equiv 0$ on $\operatorname{Supp}(m)$ and $u_{0} \not \equiv 0$ on $\partial \Omega_{0}$. So we have $\int_{\partial \Omega} m\left|u_{0}\right|^{p} d \sigma=0$ and then $v_{0}=\frac{u_{0}}{\left\|u_{0}\right\|_{L^{p}(\partial \Omega)}^{1 / p}}$ belongs to the minimizing set $\mathcal{A}$. As a consequence $\beta(V, m)<\infty$. Besides, using the definitions of $\beta(V, m)$ and $\mu_{1}(\lambda)$ we get

$$
\beta(V, m) \geq \mu_{1}(\lambda) \text { for every } \lambda \in \mathbb{R},
$$

and we infer that $\mu_{1}(\lambda)$ is bounded from above. Arguing as above, we deduce that $\varphi_{n}$ converges to some $\varphi_{0}$ weakly in $W^{1, p}(\Omega)$ and strongly in $L^{p}(\Omega)$ and in $L^{p}(\partial \Omega)$. Moreover we have

$$
\begin{aligned}
\beta(V, m) & \geq \lim _{n \rightarrow+\infty} \mu_{1}\left(\lambda_{n}\right) \\
& \geq E_{V}\left(\varphi_{0}\right)-\lim _{n \rightarrow+\infty} \lambda_{n} \int m\left|\varphi_{n}\right|^{p} d \sigma
\end{aligned}
$$


Using (3.13), we conclude that

$$
\int_{\partial \Omega} m\left|\varphi_{n}\right|^{p} d \sigma \rightarrow \int_{\partial \Omega} m\left|\varphi_{0}\right|^{p} d \sigma=0
$$

It follows that $\varphi_{0}$ is admissible in the definition of $\beta(V, m)$, and still from (3.13) we have

$$
\beta(V, m) \geq E_{V}\left(\varphi_{0}\right) \geq \beta(V, m)
$$

and the result follows.

(b) If $m^{-} \not \equiv 0$ on $\partial \Omega$ then it follows from (1), (2) and the continuity of the map $\lambda \mapsto \mu_{1}(\lambda)$, that $\mu_{1}$ is bounded from above, so that $\sup \mu_{1}(\lambda)$ is achieved at some $\lambda_{0}$ that satisfies $\lambda \in \mathbb{R}$

$$
0=\mu_{1}^{\prime}\left(\lambda_{0}\right)=-\int_{\partial \Omega} m\left|\varphi_{\lambda_{0}}\right|^{p} d \sigma
$$

Consequently $\varphi_{\lambda_{0}}$ is admissible in the definition of $\beta(V, m)$ and thus

$$
\beta(V, m) \leq E_{V}\left(\varphi_{\lambda_{0}}\right)=\mu_{1}\left(\lambda_{0}\right)=\sup _{\lambda \in \mathbb{R}} \mu_{1}(\lambda) .
$$

Then the conclusion follows.

We are now in position to give the following result.

Theorem 3.3. Let $\Omega \subset \mathbb{R}^{N}$ be a bounded smooth domain of class $C^{2, \alpha}, 0<\alpha<$ 1 , with boundary $\partial \Omega$. Assume that $V$ and $m$ satisfy $\left(H_{V}\right)$ and $\left(H_{m}\right)$ respectively and that $\lambda_{1}^{D}(V)>0$. We consider the following eigenvalue problem

$$
\begin{cases}\Delta_{p} u=V|u|^{p-2} u & \text { in } \Omega \\ |\nabla u|^{p-2} \frac{\partial u}{\partial \nu}=\lambda m(x)|u|^{p-2} u & \text { in } \partial \Omega\end{cases}
$$

(1) If $m^{-} \equiv 0$ then there exists a principal eigenvalue of (3.14) if and only if $\beta(V, m)>0$. In this case the principal eigenvalue is unique and it is defined by

$$
\lambda_{1}(V, m)=\inf _{u \in \mathcal{M}^{+}} E_{V}(u)
$$

where

$$
\mathcal{M}^{+} \stackrel{\text { def }}{=}\left\{u \in W^{1, p}(\Omega): \int_{\partial \Omega} m(x)|u|^{p} d \sigma=1\right\} .
$$

(2) If $m^{-} \not \equiv 0$ then there exists a principal eigenvalue of (3.14) if and only if $\beta(V, m) \geq 0$. More precisely:

(a) If $\beta(V, m)>0$ then (3.14) admits exactly two principal eigenvalues $\lambda_{-1}(V, m)<\lambda_{1}(V, m)$, with $\lambda_{1}(V, m)$ defined above and

$$
\lambda_{-1}(V, m)=-\inf _{u \in \mathcal{M}^{-}} E_{V}(u)
$$

where

$$
\mathcal{M}^{-} \stackrel{\text { def }}{=}\left\{u \in W^{1, p}(\Omega): \int_{\partial \Omega} m|u|^{p} d \sigma=-1\right\} .
$$


(b) If $\beta(V, m)=0$ then (3.14) has an unique principal eigenvalue $\lambda_{1}(V, m)$ given by

$$
\lambda_{1}(V, m)=\inf _{u \in \mathcal{M}^{+}} E_{V}(u)=-\inf _{u \in \mathcal{M}^{-}} E_{V}(u) .
$$

These infima are not achieved. Moreover any function $u \not \equiv 0$ in $W^{1, p}(\Omega)$ satisfying

$$
E_{V}(u)=\int_{\partial \Omega} m(x)|u|^{p} d \sigma=0
$$

is an eigenfunction associated to $\lambda_{1}(V, m)$.

Proof. We only sketch it since it is rather similar to the proof of Theorem 7 in $[8]$.

(1) From Proposition 3.2, it is clear that the condition $\beta(V, m)>0$ is necessary and sufficient for the function $\mu_{1}(\lambda)$ to vanish at some unique $\lambda_{1}$. Moreover from the definition of $\mu_{1}(\lambda)$, it follows that

$$
E_{V}(u)=\int_{\Omega}\left(|\nabla u|^{p}+V(x)|u|^{p}\right) d x \geq \lambda_{1} \int_{\partial \Omega} m(x)|u|^{p} d \sigma,
$$

for all $u \in W^{1, p}(\Omega)$ and equality holds if $u=\varphi_{\lambda_{1}}$. This implies (3.15).

(2) We know from Proposition 3.2, that $\mu_{1}$ is concave, differentiable and $\lim _{\lambda \rightarrow \pm \infty} \mu_{1}(\lambda)=-\infty$. So if $m^{-} \not \equiv 0$ and $\beta(V, m)>0$, then $\mu_{1}$ vanishes twice, let's say in $\lambda_{-1}(V, m)<\lambda_{1}(V, m)$ and we deduce (3.16).

If $m^{-} \not \equiv 0$ and $\beta(V, m)=0$ then $\mu_{1}\left(\lambda_{0}\right)=0$ at some $\lambda_{0}$ providing a principal eigenvalue of (3.14). By Proposition 3.2 we deduce that $\lambda_{0}$ is unique. Moreover $\lambda_{0}$ is principal and is a global maximum point of $\mu_{1}$, so

$$
\mu_{1}^{\prime}\left(\lambda_{0}\right)=-\int_{\partial \Omega} m\left|\varphi_{\lambda_{0}}\right|^{p} d \sigma=0
$$

We will prove that $\lambda_{0}=\inf _{\mathcal{M}^{+}} E_{V}(u)$. Since $\mu_{1}\left(\lambda_{0}\right)=0$, one observes that

$$
\mu_{1}\left(\lambda_{0}\right)=0 \leq E_{V}(u)-\lambda_{0} \int_{\partial \Omega} m|u|^{p} d \sigma, \quad \forall u \in W^{1, p}(\Omega) .
$$

In particular

$$
E_{V}(u) \geq \lambda_{0}, \quad \forall u \in \mathcal{M}^{+}
$$

and consequently $\inf _{u \in \mathcal{M}^{+}} E_{V}(u) \geq \lambda_{0}$.

Consider now the sequence $u_{n} \in \mathcal{M}^{+}$given by

$$
u_{n}=\frac{\varphi_{\lambda_{0}}+\frac{\Psi}{n}}{\left[\int_{\partial \Omega} m\left|\varphi_{\lambda_{0}}+\frac{\Psi}{n}\right|^{p} d \sigma\right]^{1 / p}}
$$

where $0<\Psi \in C^{\infty}(\partial \Omega)$ such that $\int_{\partial \Omega} m|\Psi|^{p} d \sigma>0$. One can easily see that $\int_{\partial \Omega} m\left|\varphi_{\lambda_{0}}+\frac{\Psi}{n}\right|^{p} d \sigma>0$ for $n$ large enough. Moreover, by mean value theorem, for such $n$ we can find $0<t_{n}, s_{n}<\frac{1}{n}$ such that

$$
E_{V}\left(\varphi_{\lambda_{0}}+\frac{\Psi}{n}\right)=\frac{1}{n}\left\langle E_{V}^{\prime}\left(\varphi_{\lambda_{0}}+t_{n} \Psi\right), \Psi\right\rangle
$$


and

$$
\int_{\partial \Omega} m\left|\varphi_{\lambda_{0}}+\frac{\Psi}{n}\right|^{p} d \sigma=\frac{p}{n} \int_{\partial \Omega} m\left|\varphi_{\lambda_{0}}+s_{n} \Psi\right|^{p-1} \Psi d \sigma
$$

Hence

$$
E_{V}\left(u_{n}\right)=\frac{E_{V}\left(\varphi_{\lambda_{0}}+\frac{\Psi}{n}\right)}{\int_{\partial \Omega}\left|\varphi_{\lambda_{0}}+\frac{\Psi}{n}\right|^{p} d \sigma}=\frac{\left\langle E_{V}^{\prime}\left(\varphi_{\lambda_{0}}+t_{n} \Psi\right), \Psi\right\rangle}{p \int_{\partial \Omega} m\left|\varphi_{\lambda_{0}}+s_{n} \Psi\right|^{p-1} \Psi d \sigma} \rightarrow \lambda_{0}
$$

as $n \rightarrow \infty$. On the other hand, since $\mu_{1}^{\prime}\left(\lambda_{0}\right)=-\int_{\partial \Omega} m \varphi_{\lambda_{0}}^{p} d \sigma=0$ we deduce that $\lambda_{0}$ is not achieved. A similar argument holds for $u \in \mathcal{M}^{-}$ and $\Psi<0$ with $\int_{\partial \Omega} m \Psi^{p} d \sigma<0$ and $\int_{\partial \Omega} m \varphi_{\lambda_{0}}^{p-1} \Psi d \sigma<0$. Thus we also have $\lambda_{0}=\inf _{u \in \mathcal{M}^{-}} E_{V}(u)$.

Finally if $u$ is such that

$$
E_{V}(u)=0=\int_{\partial \Omega} m|u|^{p} d \sigma
$$

after $L^{p}(\partial \Omega)$-normalization, one has $\int_{\partial \Omega}|u|^{p} d \sigma=1$ and

$$
\sup _{\lambda \in \mathbb{R}} \mu_{1}(\lambda)=0=E_{V}(u)=\Phi_{\lambda_{0}}(u) \geq \mu_{1}\left(\lambda_{0}\right)=0 .
$$

So $u$ achieves $\mu_{1}\left(\lambda_{0}\right)$, consequently $u=c \varphi_{\lambda_{0}}$ for some constant $c$ and the result follows.

\section{Non-principal eigenvalues}

In this section, we turn to the search of a first non-principal eigenvalue of (3.14) which is greater than $\lambda_{1}(V, m)$ when $\beta(V, m) \geq 0$. For this purpose we will apply a mountain pass theorem along a family of paths depending on the normalized eigenfunction (when it exists) associated to the principal eigenvalue. This approach is used for the standard boundary condition problems (Dirichlet and Neumann) in $[3,4,8]$.

We denote by $\tilde{E}_{V}$ the restriction of $E_{V}$ to the manifold $\mathcal{M}^{+}$. For any $u \in \mathcal{M}^{+}$, the tangent space of $\mathcal{M}^{+}$at $u$, denoted by $T_{u} \mathcal{M}^{+}$, is the set

$$
T_{u} \mathcal{M}^{+} \stackrel{\text { def }}{=}\left\{w \in \mathcal{M}^{+}: \int_{\partial \Omega} m|u|^{p-2} u w=0\right\} .
$$

Let us also denote by $\varphi_{1} \in \mathcal{M}^{+}$the positive eigenfunction associated to $\lambda_{1}(V, m)$ when $\beta(V, m)>0$ and by $\varphi_{0}$ the $L^{p}(\partial \Omega)$-normalized positive eigenfunction associated to $\lambda_{1}(V, m)$ such that $\int_{\partial \Omega} m \varphi_{0}^{p} d \sigma=0$ when $\beta(V, m)=0$. We recall that the infimum $\lambda_{1}(V, m)$ is not achieved when $\beta(V, m)=0$, so we will consider separately the cases $\beta(V, m)>0$ and $\beta(V, m)=0$.

Proposition 4.1. Assume that $\beta(V, m)>0$ and $\lambda_{1}^{D}(V)>0$. Then the functional $\tilde{E}_{V}$ satisfies the Palais-Smale condition.

Proof. Let $\left(u_{n}\right)$ be a Palais-Smale sequence in $\mathcal{M}^{+}$for $\tilde{E}_{V}$. So

$$
E_{V}\left(u_{n}\right)=\int_{\Omega}\left|\nabla u_{n}\right|^{p}+\int_{\Omega} V\left|u_{n}\right|^{p} \rightarrow c
$$


for some constant $c$, and there exists $\varepsilon_{n} \rightarrow 0$ such that

$$
\left.\left|\int_{\Omega}\right| \nabla u_{n}\right|^{p-2} \nabla u_{n} \cdot \nabla \xi d x+\int_{\Omega} V\left|u_{n}\right|^{p-2} u_{n} \xi d x \mid \leq \varepsilon_{n}\|\xi\| .
$$

for all $\xi \in T_{u_{n}} \mathcal{M}^{+}$. Here $\|\xi\|^{p}=\int_{\Omega}|\nabla \xi|^{p}+\int_{\partial \Omega}|\xi|^{p}$ is a norm equivalent to the usual norm of $W^{1, p}(\Omega)$.

For any $v \in W^{1, p}(\Omega)$, it is clear that

$$
a_{n}(v)=v-\left(\int_{\partial \Omega} m\left|u_{n}\right|^{p-2} u_{n} v d \sigma\right) u_{n} \in T_{u_{n}} \mathcal{M}^{+}
$$

Hence, taking $\xi=a_{n}(v)$ in (4.1), we get

$$
\begin{aligned}
& \left|\left\langle E_{V}^{\prime}\left(u_{n}\right), v\right\rangle-\left(\int_{\partial \Omega} m\left|u_{n}\right|^{p-2} u_{n} v d \sigma\right) E_{V}\left(u_{n}\right)\right| \\
& \quad \leq \varepsilon_{n}\left\|v-\left(\int_{\partial \Omega} m\left|u_{n}\right|^{p-2} u_{n} v d \sigma\right) u_{n}\right\| .
\end{aligned}
$$

We claim that $u_{n}$ remains bounded in $W^{1, p}(\Omega)$. Indeed assume by contradiction that $u_{n}$ is unbounded. Then, up to a subsequence, $v_{n}=\frac{u_{n}}{\left\|u_{n}\right\|}$ converges to some $v_{0}$ weakly in $W^{1, p}(\Omega)$ and strongly in $L^{p}(\Omega)$ and in $L^{p}(\partial \Omega)$. Thus, passing to the limit, we deduce that $\int_{\partial \Omega} m\left|v_{0}\right|^{p} d \sigma=0$ and

$$
\int_{\Omega}\left|\nabla v_{0}\right|^{p} d x+\int_{\Omega} V(x)\left|v_{0}\right|^{p} d x \leq \liminf E_{V}\left(v_{n}\right)=\liminf \frac{E_{V}\left(u_{n}\right)}{\left\|u_{n}\right\|^{p}}=0 .
$$

Furthermore

$$
\begin{aligned}
1+\int_{\Omega} V(x)\left|v_{0}\right|^{p} d x & =\lim _{n \rightarrow+\infty}\left[1+\int_{\Omega} V(x)\left|v_{n}\right|^{p}\right] d x \\
& =\lim _{n \rightarrow+\infty}\left[\int_{\Omega}\left|\nabla v_{n}\right|^{p} d x+\int_{\partial \Omega}\left|v_{n}\right|^{p} d \sigma+\int_{\Omega} V(x)\left|v_{n}\right|^{p}\right] d x \\
& =\lim _{n \rightarrow+\infty}\left[E_{V}\left(v_{n}\right)+\int_{\partial \Omega}\left|v_{n}\right|^{p} d \sigma\right] \\
& =\int_{\partial \Omega}\left|v_{0}\right|^{p} d \sigma
\end{aligned}
$$

and consequently $v_{0} \not \equiv 0$ in $\Omega$. If $v_{0} \equiv 0$ in $\partial \Omega$ then $v_{0} \in W_{0}^{1, p}(\Omega)$ and we deduce from (4.3) that

$$
\lambda_{1}^{D}(V) \int_{\Omega}\left|v_{0}\right|^{p} d x \leq \int_{\Omega}\left|\nabla v_{0}\right|^{p} d x+\int_{\Omega} V\left|v_{0}\right|^{p} d x \leq 0,
$$

a contradiction since $\lambda_{1}^{D}(V)>0$. Hence $v_{0} \not \equiv 0$ in $\partial \Omega$ and $\frac{v_{0}}{\left\|v_{0}\right\|_{L^{p}(\partial \Omega)}}$ is an admissible function in the definition of $\beta(V, m)$ and consequently

$$
\beta(V, m) \leq E_{V}\left(\frac{v_{0}}{\left\|v_{0}\right\|_{L^{p}(\partial \Omega)}}\right) \leq 0 .
$$

This again yields to a contradiction with the assumption $\beta(V, m)>0$ and we conclude that $u_{n}$ is a bounded sequence in $W^{1, p}(\Omega)$. Up to a subsequence 
$u_{n} \rightarrow u_{0}$ weakly in $W^{1, p}(\Omega)$ and strongly in $L^{p}(\Omega)$ and in $L^{p}(\partial \Omega)$. Choosing $v=u_{n}-u_{0}$ in (4.2) and passing to the limit we obtain

$$
\lim _{n \rightarrow+\infty} \int_{\Omega}\left|\nabla u_{n}\right|^{p-2} \nabla u_{n} \nabla\left(u_{n}-u_{0}\right) d x=0 .
$$

Applying (3.10) and Hölder inequality, one easily derives that $\nabla u_{n} \rightarrow \nabla u_{0}$ in $L^{p}(\Omega)$ and consequently $u_{n} \rightarrow u_{0}$ in $W^{1, p}(\Omega)$.

Proposition 4.2. Assume that $\beta(V, m)>0$ and $\lambda_{1}^{D}(V)>0$ and let

$$
\lambda_{2}(V, m) \stackrel{\text { def }}{=} \inf _{\gamma \in \Gamma} \max _{t \in[0,1]} E_{V}(\gamma(t))
$$

where $\Gamma=\left\{\gamma \in \mathcal{C}\left([0,1], \mathcal{M}^{+}\right): \gamma(0)=-\varphi_{1}\right.$ and $\left.\gamma(1)=\varphi_{1}\right\}$. Then $\lambda_{2}(V, m)$ is a non-principal eigenvalue of (3.14) greater than $\lambda_{1}(V, m)$.

Proof. We will first prove that the set $\Gamma$ is nonempty. Clearly it suffices to construct a path $\gamma$ in $\mathcal{M}^{+}$from $-\varphi_{1}$ to $\varphi_{1}$ such that $\int_{\partial \Omega} m|\gamma(t)|^{p} d \sigma>0$ for all $t$.

Let recall the open set $\partial \Omega^{+}=\{x \in \partial \Omega: m(x)>0\}$. One can find two disjoint compact sets $K_{1}$ and $K_{2}$ in $\partial \Omega^{+}$and construct two functions $u_{1}$ and $u_{2}$ in $\mathcal{D}\left(\partial \Omega^{+}\right)$such that

$$
\begin{array}{lll}
u_{1} \equiv 1 & \text { on } K_{1}, \quad 0 \leq u_{1} \leq 1 \quad \text { on } \partial \Omega^{+}, \\
u_{2} \equiv 1 \quad \text { on } K_{2}, \quad 0 \leq u_{2} \leq 1 \quad \text { on } \partial \Omega^{+} .
\end{array}
$$

Extending $u_{1}$ and $u_{2}$ by zero on $\partial \Omega \backslash \partial \Omega^{+}$, we get $u_{1}, u_{2} \in \mathcal{D}(\partial \Omega)$. We then set $u_{0}=u_{1}-u_{2}$ and get a function in $\mathcal{D}(\partial \Omega)$ with

$$
\int_{\partial \Omega} m\left(u_{0}^{+}\right)^{p} d \sigma>0 \text { and } \int_{\partial \Omega} m\left(u_{0}^{-}\right)^{p} d \sigma>0 .
$$

We first go from $u_{0}$ to $u_{0}^{+}$by a convex combination:

$$
\gamma_{1}(t)=u_{0}^{+}-t u_{0}^{-}, \quad t \in[0,1],
$$

then we go on from $u_{0}^{+}$to $\varphi_{1}$ through the path

$$
\gamma_{2}(t)=\left[t\left(u_{0}^{+}\right)^{p}+(1-t) \varphi_{1}^{p}\right]^{1 / p}, \quad t \in[0,1] .
$$

Moreover one has

$$
\int_{\partial \Omega} m\left|\gamma_{1}(t)\right|^{p} d \sigma=\int_{\partial \Omega} m\left|u_{0}^{+}\right|^{p} d \sigma+t^{p} \int_{\partial \Omega} m\left|u_{0}^{-}\right|^{p} d \sigma>0, \quad \forall t \in[0,1]
$$

and

$$
\int_{\partial \Omega} m\left|\gamma_{2}(t)\right|^{p} d \sigma=t \int_{\partial \Omega} m\left|u_{0}^{+}\right|^{p} d \sigma+(1-t) \int_{\partial \Omega} m\left|\varphi_{1}\right|^{p} d \sigma>0, \quad \forall t \in[0,1]
$$

Consequently, one has a path $\gamma$ joining $u_{0}$ and $\varphi_{1}$ which satisfies $\int_{\partial \Omega} m \mid \gamma$ $\left.(t)\right|^{p} d \sigma>0$ for all $t \in[0,1]$. In a similar way, we get a path $\gamma^{\prime}$ from $u_{0}$ to $-\varphi_{1}$ such that $\int_{\partial \Omega} m\left|\gamma^{\prime}(t)\right|^{p} d \sigma>0$ for all $t \in[0,1]$. Putting everything together, after normalization, we get a path on $\mathcal{M}^{+}$from $\varphi_{1}$ to $-\varphi_{1}$ and consequently $\Gamma \neq \emptyset$. 
By standard arguments, we can easily prove that $\varphi_{1}$ and $-\varphi_{1}$ minimize $\tilde{E}_{V}$ locally and strictly. Moreover $\tilde{E}_{V}$ has the mountain-pass geometry (cf. Lemma 6 of [3]), i.e. for every $\varepsilon>0$

$$
\lambda_{1}(V, m)<\inf \left\{\tilde{E}_{V}(u), u \in \mathcal{M}^{+} \cap \partial B\left( \pm \varphi_{1}, \varepsilon\right)\right\} .
$$

Consequently $\lambda_{2}(V, m)>\lambda_{1}(V, m)$. Since $\tilde{E}_{V}$ satisfies the Palais-Smale condition, a version of the mountain-pass theorem on $C^{1}$-manifolds of $[3,4]$ allows us to deduce that $\lambda_{2}(V, m)$ is an eigenvalue of (3.14).

Lemma 4.3. Assume that $\beta(V, m)>0$ and $\lambda_{1}^{D}(V)>0$. For every $d \in \mathbb{R}$, the set $\mathcal{O}=\left\{u \in \mathcal{M}^{+}, u \geq \operatorname{0andE}_{V}(u)<d\right\}$ is arcwise connected. The same conclusion holds if the condition $u \geq 0$ is replaced by $u \leq 0$.

Proof. We will first consider the sublevel set in $\mathcal{M}^{+}$

$$
\hat{\mathcal{O}}=\left\{u \in \mathcal{M}^{+}, \quad E_{V}(u)<d\right\}
$$

It is clear that $\mathcal{O} \subset \hat{\mathcal{O}}$ and $\hat{\mathcal{O}}$ is empty if $d \leq \lambda_{1}(V, m)$. So we assume that $d>\lambda_{1}(V, m)$ and we get clearly that $\varphi_{1}$ is the only critical point of $E_{V}$ which belongs to $\hat{\mathcal{O}}$. On the other hand, since $E_{V}$ is bounded below on $\mathcal{M}^{+}$and satisfy the Palais-Smale condition, we can use Lemma 14 in [3] to conclude that $\hat{\mathcal{O}}$ is arcwise connected.

Now, let $u_{1}, u_{2} \in \mathcal{O}$. Since $\mathcal{O} \subset \hat{\mathcal{O}}$ there exists a path $\gamma \in \hat{\mathcal{O}}$ going from $u_{1}$ to $u_{2}$. We then consider the path $\gamma_{2}=\left|\gamma_{1}\right|$ which clearly goes from $u_{1}$ to $u_{2}$, lies in $\mathcal{M}^{+}$and is made of nonnegative functions with $E_{V}\left(\gamma_{2}(t)\right)<d$ for all $t \in[0,1]$. Consequently $\gamma$ is a path in $\mathcal{O}$ which connects $u_{1}$ to $u_{2}$ and then $\mathcal{O}$ is arcwise connected.

Remark 4.1. As observed in [3, Remark 17], from the proof of Lemma 4.3, one has the following fact: Given $u \in \mathcal{M}^{+}$with $u \geq 0$ (resp. $u \leq 0$ ) and $E_{V}(u)<d$ for some $d$, there exists a path in $\mathcal{M}^{+}$from $u$ to $\varphi_{1}$ (resp. $u$ to $-\varphi_{1}$ ), which is made of nonnegative functions (resp. nonpositive functions) and which remains at levels $<d$. Indeed since the set $\mathcal{O}$ is arcwise connected and $\varphi_{1} \in \mathcal{O}$, it suffices to take $u=u_{1} \in \mathcal{O}$ (with $u \geq 0$ ), $u_{2}=\varphi_{1}$ and the path $\gamma_{1}$ defined in the proof of Lemma 4.3 which connects $u_{1}$ to $u_{2}$. According to the proof of Lemma 4.3, this path lies in $\mathcal{M}^{+}$and remains at levels $<d$. A similar argument holds for $u=u_{1} \in \mathcal{O}$ (with $u \leq 0$ ) and $u_{2}=-\varphi_{1}$.

One can modify a little bit the variational characterization (4.4) of $\lambda_{2}(V, m)$ by enlarging the family of paths and keep the same minimax level.

Proposition 4.4. Assume that $\beta(V, m)>0$ and $\lambda_{1}^{D}(V)>0$. One has

$$
\lambda_{2}(V, m)=\inf _{\gamma \in \Gamma_{1}} \max _{t \in[0,1]} E_{V}(\gamma(t))
$$

where

$$
\Gamma_{1} \stackrel{\text { def }}{=}\left\{\gamma \in \mathcal{C}\left([0,1], \mathcal{M}^{+}\right): \gamma(0) \leq 0 \text { and } \gamma(1) \geq 0\right\}
$$

Proof. Let us call $d$ the right hand side of (4.5). Since $\Gamma \subset \Gamma_{1}$, it is clear that $d \leq \lambda_{2}(V, m)$. Assume by contradiction that $d<\lambda_{2}(V, m)$ and take $\mu$ such that $d<\mu<\lambda_{2}(V, m)$. Choose a path $\gamma \in \Gamma_{1}$ which remains at levels $<\mu$. 
We will construct a path in $\Gamma$ which also remains at levels $<\mu$. This will contradict the definition (4.4) of $\lambda_{2}(V, m)$. To construct this path we first go from $\varphi_{1}$ to $\gamma(1)$ by using Remark 4.1, then we follow $\gamma$ from $\gamma(1)$ to $\gamma(0)$ and again using Remark 4.1 we go from $\gamma(0)$ to $-\varphi_{1}$. Since all those paths belong to $\mathcal{M}^{+}$, putting everything together we get a path $\gamma_{1}$ on $\Gamma$ which goes from $\varphi_{1}$ to $-\varphi_{1}$ and remains at levels $<\mu$, i.e. $E_{V}\left[\gamma_{1}(t)\right]<\mu<\lambda_{2}(V, m)$.

Proposition 4.5. Assume that $\beta(V, m)>0$ and $\lambda_{1}^{D}(V)>0$. Then problem (3.14) does not admit any eigenvalue in the interval $] \lambda_{1}(V, m), \lambda_{2}(V, m)[$.

Proof. Assume by contradiction that there exists an eigenvalue $\lambda$ of (3.14) with $\lambda \in] \lambda_{1}(V, m), \lambda_{2}(V, m)[$, and let $u$ be the associated eigenfunction. Then $u$ changes sign and $u$ satisfies the equation

$$
\int_{\Omega}|\nabla u|^{p-2} \nabla u \nabla v d x+\int_{\Omega} V|u|^{p-2} u v d x=\lambda \int_{\partial \Omega} m|u|^{p-2} u v d \sigma
$$

for all $v \in W^{1, p}(\Omega)$. This implies that

$$
\begin{aligned}
& E_{V}\left(u^{+}\right)=\int_{\Omega}\left|\nabla u^{+}\right|^{p} d x+\int_{\Omega} V\left(u^{+}\right)^{p} d x=\lambda \int_{\partial \Omega} m\left(u^{+}\right)^{p} d \sigma \quad \text { and } \\
& E_{V}\left(u^{-}\right)=\int_{\Omega}\left|\nabla u^{-}\right|^{p} d x+\int_{\Omega} V\left(u^{-}\right)^{p} d x=\lambda \int_{\partial \Omega} m\left(u^{-}\right)^{p} d \sigma .
\end{aligned}
$$

We claim that $\int_{\partial \Omega} m\left(u^{+}\right)^{p} d \sigma>0$ and $\int_{\partial \Omega} m\left(u^{-}\right)^{p} d \sigma>0$. Indeed

- If $\int_{\partial \Omega} m\left(u^{+}\right)^{p} d \sigma=0$ then

$$
\beta(V, m) \leq E_{V}\left(\frac{u^{+}}{\left\|u^{+}\right\|_{L^{p}(\partial \Omega)}}\right)=\frac{\lambda \int_{\partial \Omega} m\left(u^{+}\right)^{p} d \sigma}{\left\|u^{+}\right\|_{L^{p}(\partial \Omega)}^{p}}=0,
$$

a contradiction.

- If $\int_{\partial \Omega} m\left(u^{+}\right)^{p} d \sigma<0$ then $m$ is sign-changing. Thus

$$
-\lambda=E_{V}\left(\frac{u^{+}}{\left[-\int_{\partial \Omega} m\left(u^{+}\right)^{p} d \sigma\right]^{1 / p}}\right) \geq-\lambda_{-1}(V, m) \geq-\lambda(V, m)
$$

which yields again a contradiction. In like manner, we show that $\int_{\partial \Omega} m\left(u^{-}\right)^{p} d \sigma>0$. It follows that

$$
\begin{aligned}
\int_{\partial \Omega} m\left|t u+(1-t) u^{+}\right|^{p} d \sigma & =\int_{\partial \Omega} m\left|u^{+}-t u^{-}\right|^{p} d \sigma \\
& =\int_{\partial \Omega} m\left(u^{+}\right)^{p} d \sigma+t^{p} \int_{\partial \Omega} m\left(u^{-}\right)^{p} d \sigma>0 \quad \forall t \in[0,1] .
\end{aligned}
$$

Thus the path

$$
\gamma_{1}(t)=\frac{u^{+}-t u^{-}}{\left(\int_{\partial \Omega} m\left|u^{+}-t u^{-}\right|^{p} d \sigma\right)^{1 / p}}
$$

is well defined, belongs to $\mathcal{M}^{+}$, goes from $v_{1}=\frac{u^{+}}{\left(\int_{\partial \Omega} m\left(u^{+}\right)^{p} d \sigma\right)^{1 / p}}$ to $v_{2}=\frac{u}{\left(\int_{\partial \Omega} m|u|^{p} d \sigma\right)^{1 / p}}$ and satisfies $E_{V}\left[\gamma_{1}(t)\right]=\lambda$ for all $t \in[0,1]$. In a 
similar way we set the path

$$
\gamma_{2}(t)=\frac{(1-t) u^{+}-u^{-}}{\left(\int_{\partial \Omega} m\left|(1-t) u^{+}-u^{-}\right|^{p} d \sigma\right)^{1 / p}}
$$

which is a path in $\mathcal{M}^{+}$going from $v_{2}=\frac{u}{\left(\int_{\partial \Omega} m|u|^{p} d \sigma\right)^{1 / p}}$ to $v_{3}=$ $-\frac{u^{-}}{\left(\int_{\partial \Omega} m\left(u^{-}\right)^{p} d \sigma\right)^{1 / p}}$ such that $E_{V}\left[\gamma_{2}(t)\right]=\lambda$ for all $t \in[0,1]$. Hence $\gamma_{1} \cup \gamma_{2}$ provides a path going from $v_{1}$ to $v_{3}$ such that $E_{V}\left[\left(\gamma_{1} \cup \gamma_{2}\right)(t)\right]=\lambda$ for all $t \in[0,1]$. Since $v_{1} \geq 0$ and $v_{3} \leq 0$, using Remark 4.1 there are paths going from $\varphi_{1}$ to $v_{1}$ and from $v_{2}$ to $-\varphi_{1}$ which stay at levels $<\lambda$. Gluing everything together we have the desired path which belongs to $\Gamma$, going from $\varphi_{1}$ to $-\varphi_{1}$ and stays at levels $<\lambda$ which contradicts the definition of $\lambda_{2}(V, m)$ in $(4.4)$.

We assume now that $\left|\partial \Omega_{-}\right|>0$ and $\beta(V, m)=0$. It has been proved previously in Theorem 3.3, that the unique principal eigenvalue $\lambda_{1}(V, m)$ of (3.14) is simple and has a positive eigenfunction $\varphi_{0}$ such that $\int_{\partial \varphi}\left|\varphi_{0}\right|^{p} d \sigma=1$ and $\int_{\partial \varphi} m\left|\varphi_{0}\right|^{p} d \sigma=0$. Consequently $\varphi_{0}$ fails to belong to the manifold $\mathcal{M}^{+}$. Therefore the mountain-pass procedure used above, which consists of minimizing $E_{V}$ over path connecting $\varphi_{0}$ and $-\varphi_{0}$ can not be applied in the present situation since $\varphi_{0} \notin \mathcal{M}^{+}$. To bypass this difficulty, we will consider the approach of minimizing $E_{V}$ over free endpoints paths on $\mathcal{M}^{+}$going from a positive function to a negative one and staying at an energy level above $\lambda_{1}(V, m)$. This approach has been used in [4] for a Neumann problem. We aim to apply the mountain pass theorem for a $C^{1}$ functional which satisfies the PSC condition on a $C^{1}$ manifold, instead of the usual PS condition (cf. Theorem 4.1 in [4]).

Let us introduce

$$
c(V, m)=\inf _{\gamma \in \Gamma_{1}} \max _{t \in[0,1]} E_{V}(\gamma(t))
$$

where $\Gamma_{1}$ is defined in (4.6).

We will prove the following result

Lemma 4.6. Assume $\left|\partial \Omega_{-}\right|>0, \lambda_{1}^{D}(V)>0$ and $\beta(V, m)=0$. Then $c(V, m)>$ $\lambda_{1}(V, m)$.

Proof. An easy adaptation of the proof of Proposition 18 in [8].

Lemma 4.7. Assume that $\left|\partial \Omega_{-}\right|>0, \lambda_{1}^{D}(V)>0$ and $\beta(V, m)=0$. There exists $u_{1} \in \mathcal{M}^{+}$such that $u_{1} \geq 0$ and $E_{V}\left(u_{1}\right)<c(V, m)$.

Proof. Since $\beta(V, m)=0$, one takes for $n$ sufficiently large $u_{1}=v_{n} \geq 0$, where $v_{n}$ is defined in (3.18). Indeed $E_{V}\left(v_{n}\right) \rightarrow \lambda_{0}=\lambda_{1}(V, m)<c(V, m)$, as $n \rightarrow \infty$, so that $E_{V}\left(u_{1}\right)=E_{V}\left(v_{n}\right)<c(V, m)$ for $n$ sufficiently large.

Proposition 4.8. Assume that $\lambda_{1}^{D}(V)>0$ and $\beta(V, m)=0$. Then $E_{V}$ satisfies the $(P S C)_{c}$ condition on $\mathcal{M}^{+}$for every $c>\lambda_{1}(V, m)$. 
Proof. Let $\left(u_{n}\right)$ be a $(P S C)_{c}$ sequence in $\mathcal{M}^{+}$for $\tilde{E}_{V}$ for $c>\lambda_{1}(V, m)$, i.e. there exists a sequence $\varepsilon_{n} \rightarrow 0$ such that

(i) $E_{V}\left(u_{n}\right)=\int_{\Omega}\left|\nabla u_{n}\right|^{p}+\int_{\Omega} V\left|u_{n}\right|^{p} \longrightarrow c$

(ii) $\left|\left\langle E_{V}^{\prime}\left(u_{n}\right), \xi\right\rangle\right| \leq \frac{\varepsilon_{n}}{1+\left\|u_{n}\right\|}\|\xi\| \quad$ for all $\xi \in T_{u_{n}} \mathcal{M}^{+}$.

where $\|u\|^{p}=\int_{\Omega}|\nabla u|^{p}+\int_{\partial \Omega}|u|^{p} d \sigma$ is a norm equivalent to the standard one in $W^{1, p}(\Omega)$.

Arguing as in the proof of Proposition 4.1, we assume that $u_{n}$ is unbounded and we set $v_{n}=\frac{u_{n}}{\left\|u_{n}\right\|}$. Up to a subsequence, $v_{n}$ converges to some $v_{0}$ weakly in $W^{1, p}$ and strongly in $L^{p}(\Omega)$ and in $L^{p}(\partial \Omega)$. We then choose

$$
w=a_{n}\left(v_{n}-v_{0}\right)=\left(v_{n}-v_{0}\right)-\left(\int_{\partial \Omega} m\left|u_{n}\right|^{p-2} u_{n}\left(v_{n}-v_{0}\right)\right) u_{n}
$$

in (ii) and divide by $\left\|u_{n}\right\|^{p-1}$ to get

$$
\begin{aligned}
& \left|\left\langle E_{V}^{\prime}\left(v_{n}\right), v_{n}-v_{0}\right\rangle-\left(\int_{\partial \Omega} m\left|v_{n}\right|^{p-2} v_{n}\left(v_{n}-v_{0}\right)\right) E_{V}\left(u_{n}\right)\right| \\
& \quad \leq \frac{\varepsilon_{n}\left\|u_{n}\right\|}{1+\left\|u_{n}\right\|}\left\|\frac{v_{n}-v_{0}}{\left\|u_{n}\right\|^{p}}-\left(\int_{\partial \Omega} m\left|v_{n}\right|^{p-2} v_{n}\left(v_{n}-v_{0}\right) d \sigma\right) v_{n}\right\|
\end{aligned}
$$

Letting $n \rightarrow \infty$ and using (3.10) and Hölder inequality, we deduce that $v_{n} \rightarrow v_{0}$ in $W^{1, p}(\Omega)$. Hence $E_{V}\left(v_{0}\right)=0$ and $\int_{\partial \Omega} m\left|v_{0}\right|^{p} d \sigma=0$ so that $v_{0}=d \varphi_{0}$ for some constant $d \neq 0$ (where $\varphi_{0}$ is the positive $L^{p}(\partial \Omega$ )-normalized eigenfunction associated to $\left.\lambda_{1}(V, m)\right)$. Furthermore, passing to the limit in the equation ( iii), we conclude that $c$ is an eigenvalue for (3.14) with a sign-constant function $v_{0}$ as associated eigenfunction, a contradiction since $\lambda_{1}(V, m)$ is the unique principal eigenvalue of (3.14) (cf. Theorem 3.3).

Then $u_{n}$ is bounded and the argument used in the end of the proof of Proposition 4.1 can be easily applied here to get that $u_{n}$ converges strongly in $W^{1, p}(\Omega)$, up to a subsequence.

Theorem 4.9. Assume that $\lambda_{1}^{D}(V)>0$ and $\beta(V, m)=0$. Let

$$
\bar{\lambda}_{2}(V, m)=\inf _{\gamma \in \Gamma_{0}} \max _{t \in[0,1]} E_{V}(\gamma(t)) .
$$

where $\Gamma_{0}=\left\{\gamma \in \mathcal{C}\left([0,1], \mathcal{M}^{+}\right): \gamma(0)=u_{1}\right.$ and $\left.\gamma(1)=-u_{1}\right\}$ for every $0 \leq$ $u_{1} \in \mathcal{M}^{+}$such that $E_{V}\left(u_{1}\right)<c(V, m)$. Then $\bar{\lambda}_{2}(V, m)$ is an eigenvalue of (3.14). Moreover $\bar{\lambda}_{2}(V, m)=c(V, m)$ and $\bar{\lambda}_{2}(V, m)$ is the first non-principal eigenvalue of (3.14).

Proof. The fact that $\bar{\lambda}_{2}(V, m)$ is an eigenvalue of (3.14) is a consequence of [4, Theorem 4.1], since $E_{V}$ satisfies the $(P S C)_{c}$ condition on the manifold $\mathcal{M}^{+}$ (with $c>\lambda_{1}(V, m)$ ) and $\Gamma_{0} \neq \emptyset$.

Next we will prove that $\bar{\lambda}_{2}(V, m)=c(V, m)$. Using the definition of $c(V, m)$ in (4.7) and the fact that $\Gamma_{0} \subset \Gamma_{1}$, we deduce that $\bar{\lambda}_{2}(V, m) \geq c(V, m)$. To prove the converse inequality, let $\epsilon>0$ and $\gamma_{\epsilon} \in \Gamma_{1}$ such that

$$
\max _{u \in \gamma_{\epsilon}([0,1])} E_{V}(u)<c(V, m)+\epsilon .
$$


By Lemma 4.3 there exists a path $\gamma_{1}$ in $\mathcal{M}^{+}$joining $\gamma_{\epsilon}(1)$ and $u_{1}$, made of nonnegative functions and such that

$$
\max _{u \in \gamma_{1}([0,1])} E_{V}(u)<c(V, m)+\epsilon .
$$

Similarly there exists a path $\gamma_{2}$ in $\mathcal{M}^{+}$joining $\gamma_{\epsilon}(0)$ and $-u_{1}$, made of nonpositive functions and such that

$$
\max _{u \in \gamma_{1}([0,1])} E_{V}(u)<c(V, m)+\epsilon .
$$

Gluing together $\gamma_{1}, \gamma_{2}$ and $\gamma_{\epsilon}$, one gets a path in $\mathcal{M}^{+}$joining $u_{1}$ and $-u_{1}$ such that $E_{V}$ remains at levels $<c(V, m)+\epsilon$, along this path. This implies that $\bar{\lambda}_{2}(V, m) \leq c(V, m)+\epsilon$. Since $\epsilon>0$ is arbitrary, the conclusion follows.

Finally, we can adapt the proof of Proposition 4.5 to this case and conclude that $\bar{\lambda}_{2}(V, m)$ is the first non-principal eigenvalue of (3.14).

\section{Related results}

The tools used to establish the above results can be easily adapted for the following Dirichlet-Steklov boundary eigenvalue problem

$$
\begin{cases}-\Delta_{p} u+V(x)|u|^{p-2} u=0 & \text { in } \Omega \\ |\nabla u|^{p-2} \frac{\partial u}{\partial \nu}=\lambda m(x)|u|^{p-2} u+\mu|u|^{p-2} u & \text { on } \Gamma_{1} \\ u=0 & \text { on } \Gamma_{2}\end{cases}
$$

where $\Omega$ is a bounded smooth domain of $C^{2, \alpha}$ ( with $0<\alpha<1$ ) in $\mathbb{R}^{N}, \nu$ the outward unit normal on the boundary $\partial \Omega$ splits up in two sets $\Gamma_{1}$ and $\Gamma_{2}$ which are connected and closed $(n-1)$-dimensional manifolds; $V$ and $m$ are indefinite functions; $\lambda$ and $\mu$ are constants parameters. This problem was considered in [13] in the linear case, i.e. $p=2$ with $m \equiv 1$ and $\mu=0$.

The functional framework for the problem (5.1) is the space $E(\Omega)$ which is defined as

$$
E(\Omega) \stackrel{\text { def }}{=}\left\{u \in W^{1, p}(\Omega): u \equiv 0 \mathrm{on} \Gamma_{2} \text { in the sense of the traces }\right\} .
$$

One can show that

$$
\|u\|_{E(\Omega)}^{p}=\int_{\Omega}|\nabla u|^{p} d x+\int_{\Gamma_{2}}|u|^{p} d \sigma=\int_{\Omega}|\nabla u|^{p} d x
$$

is a norm on $E(\Omega)$ which is equivalent to the standard norm of $W^{1, p}(\Omega)$ (cf. $[9,29])$.

The proof of the following results can be easily adapted from that of Theorem 3.1 and Theorem 3.3 respectively. We then generalize the result of [13] to the nonlinear case with indefinite weight on the boundary.

Theorem 5.1. Assume that $V \in L^{\infty}(\Omega)$ and $m \in C^{r}(\partial \Omega)$ (for some $r \in(0,1)$ ) are indefinite functions. Then for any $\lambda \in \mathbb{R},(5.1)$ admits a principal eigenvalue $\mu_{1}=\mu_{1}(\lambda)$ if and only if

$$
\lambda_{1}^{D}(V)>0
$$


where $\lambda_{1}^{D}(V)$ stands for the principal Dirichlet eigenvalue of (2.1). Moreover $\mu_{1}(\lambda)$ is simple and it is the unique principal eigenvalue of (5.1) and it is defined by

$$
\mu_{1}(\lambda) \stackrel{\text { def }}{=} \inf _{u \in E_{1}}\left\{E_{V}(u)-\lambda \int_{\Gamma_{1}} m(x)|u|^{p} d \sigma\right\}
$$

where

$$
E_{1}=\left\{u \in E(\Omega): \int_{\Gamma_{1}}|u|^{p} d \sigma=1\right\}
$$

Proof. We only sketch it since it is an easy adaptation of the proof of Theorem 3.1. The condition $\lambda_{1}^{D}(V)>0$ is necessary since it is consequence of Theorem 2 of [12]. Let prove that this condition is sufficient. We have only to prove that the functional

$$
J_{\lambda}(u)=E_{V}(u)-\lambda \int_{\Gamma_{1}} m(x)|u|^{p} d \sigma
$$

is coercive. Indeed assume that $\lambda_{1}^{D}(V)>0$ and that there exists a sequence $\left(u_{n}\right)$ of $E_{1}$ such that $\int_{\Omega}\left|\nabla u_{n}\right|^{p} d x \rightarrow+\infty$ and $J_{\lambda}\left(u_{n}\right) \leq K$ (for some constant $K)$. Following the arguments used in the proof of Theorem 3.1, we deduce that $\int_{\Omega}\left|u_{n}\right|^{p} d x \rightarrow+\infty$. Then setting $v_{n}=\frac{u_{n}}{t_{n}}$, where $t_{n}=\left(\int_{\Omega}\left|u_{n}\right|^{p} d x\right)^{\frac{1}{p}}$, we get that $v_{n}$ converges to some $v$ weakly in $E(\Omega)$, strongly in $L^{p}(\Omega)$ and in $L^{p}\left(\Gamma_{1}\right)$. Consequently $\int_{\Omega}|v|^{p} d x=1$ and $\int_{\Gamma_{1}}|v|^{p} d \sigma=0$ and then $v \not \equiv 0$ in $\Omega$ and $v \in W_{0}^{1, p}(\Omega)$ (since $v \in E(\Omega)$ ). Finally we use the variational characterization of $\lambda_{1}^{D}(V)$ and the fact that $J_{\lambda}\left(u_{n}\right)$ is bounded to deduce that

$$
\lambda_{1}^{D}(V)=\lambda_{1}(V) \int_{\Omega}|v|^{p} d x \leq E_{V}(u) \leq \liminf J_{\lambda}\left(u_{n}\right) \leq 0,
$$

a contradiction with the assumption on $\lambda_{1}^{D}(V)$.

Theorem 5.2. Assume that $V \in L^{\infty}(\Omega)$ and $m \in C^{r}(\partial \Omega)$ (for some $r \in(0,1)$ ) are indefinite functions such that $m^{+} \not \equiv 0$ on $\Gamma_{1}$ and $\lambda_{1}^{D}(V)>0$.

We consider the following Dirichlet-Steklov boundary eigenvalue problem

$$
\begin{cases}\Delta_{p} u=V(x)|u|^{p-2} u & \text { in } \Omega \\ |\nabla u|^{p-2} \frac{\partial u}{\partial \nu}=\lambda m(x)|u|^{p-2} u & \text { on } \Gamma_{1} \\ u=0 & \text { on } \Gamma_{2}\end{cases}
$$

and define the following real

$$
\begin{gathered}
\Lambda(V, m)=\infty \quad \text { if } m>0 \text { on } \Gamma_{1} \\
\Lambda(V, m) \stackrel{\text { def }}{=} \inf \left\{E_{V}(u): \int_{\Gamma_{1}}|u|^{p} d \sigma=1\right. \\
\left.\quad \text { and } \int_{\Gamma_{1}} m(x)|u|^{p} d \sigma=0\right\} \quad \text { if }\left|\Gamma_{1}^{+}\right|<\left|\Gamma_{1}\right| .
\end{gathered}
$$

with $\Gamma_{1}^{+}=\left\{x \in \Gamma_{1}: m(x)>0\right\}$ 
(1) If $m^{-} \equiv 0$ then there exists a principal eigenvalue of (5.3) if and only if $\Lambda(V, m)>0$. In this case the principal eigenvalue is unique and it is defined by

$$
\lambda_{1}(V, m)=\inf _{u \in \mathcal{M}^{+}} \int_{\Omega}\left(|\nabla u|^{p}+V(x)|u|^{p}\right) .
$$

where

$$
\mathcal{M}^{+} \stackrel{\text { def }}{=}\left\{u \in E(\Omega): \int_{\Gamma_{1}} m(x)|u|^{p} d \sigma=1\right\} .
$$

(2) If $m^{-} \not \equiv 0$ then there exists a principal eigenvalue of (5.3) if and only if $\Lambda(V, m) \geq 0$. More precisely:

(a) If $\Lambda(V, m)>0$ then (5.3) admits exactly two principal eigenvalues $\lambda_{-1}(V, m)<\lambda_{1}(V, m)$, with $\lambda_{1}(V, m)$ defined above and

$$
\lambda_{-1}(V, m)=-\inf _{u \in \mathcal{M}^{-}} \int_{\Omega}\left(|\nabla u|^{p}+V(x)|u|^{p}\right)
$$

where

$$
\mathcal{M}^{-} \stackrel{\text { def }}{=}\left\{u \in E(\Omega): \int_{\Gamma_{1}} m|u|^{p} d \sigma=-1\right\} .
$$

(b) If $\Lambda(V, m)=0$ then (5.3) has an unique principal eigenvalue $\lambda_{1}(V, m)$ given by

$$
\lambda_{1}(V, m)=\inf _{u \in \mathcal{M}^{+}} E_{V}(u)=-\inf _{u \in \mathcal{M}^{-}} E_{V}(u) .
$$

These infima are not achieved. Moreover any function $u \not \equiv 0$ in $E(\Omega)$ satisfying

$$
E_{V}(u)=\int_{\Gamma_{1}} m(x)|u|^{p} d \sigma=0
$$

is an eigenfunction associated to $\lambda_{1}(V, m)$.

Proof. An easy adaptation of the proofs of Proposition 3.2 and Theorem 3.3.

\section{Acknowledgments}

The authors wish to thank the referees for their interesting remarks, comments and suggestions which contributed to the quality of the paper.

\section{Appendix: regularity results on eigenfunction}

In this section, some properties of weak solutions of (1.1) are proved 
Theorem 6.1. (Boundedness for eigenfunctions) Let $\Omega$ be a bounded domain in $\mathbb{R}^{N}$ with $C^{1}$ boundary $\partial \Omega$. Let $(u, \mu)$ be a weak eigensolution of

$$
\begin{cases}-\Delta_{p} u=V(x)|u|^{p-2} u & \text { in } \Omega \\ |\nabla u|^{p-2} \frac{\partial u}{\partial \nu}=(\lambda m(x)+\mu)|u|^{p-2} u & \text { on } \partial \Omega\end{cases}
$$

where $V \in L^{\infty}(\Omega), m \in L^{\infty}(\partial \Omega)$ with $m^{+} \not \equiv 0, \lambda$ a real parameter and $\mu$ an eigenvalue. Then $u \in L^{\infty}(\Omega)$.

Proof. We borrow some ideas from [18] for an analogous result.

By Sobolev's embedding theorem, it suffices to consider the case $N \geq p$. Let us assume first that $u \geq 0$. For $M>0$ we define $v_{M}(x)=\min \{u(x), M\}$. Then $v_{M} \in W^{1, p}(\Omega) \cap L^{\infty}(\Omega)$ and consequently

$$
\varphi(x)=\left[v_{M}(x)\right]^{k p+1} \in W^{1, p}(\Omega) \cap L^{\infty}(\Omega) \text { for } \quad k>0 .
$$

Using $\varphi$ a test function in (3.1), one obtains

$$
\begin{aligned}
& (1+k p) \int_{\Omega}|\nabla u|^{p-2} \nabla v_{M} \cdot v_{M}^{k p} d x=-\int_{\Omega} V|u|^{p-2} u\left(v_{M}\right)^{k p+1} d x \\
& \quad+\int_{\partial \Omega}(\lambda m+\mu)|u|^{p-2} u(v)^{k p+1} d \sigma
\end{aligned}
$$

which implies

$$
\begin{aligned}
\frac{k p+1}{(k+1)^{p}} \int_{\Omega}\left|\nabla\left(v_{M}^{k+1}\right)\right|^{p} d x \leq & \|V\|_{\infty} \int_{\Omega}\left(v_{M}\right)^{p(k+1)} d x+\left[|\lambda|\|m\|_{\infty}+|\mu|\right] \\
& \times \int_{\partial \Omega}\left(v_{M}\right)^{p(k+1)} d \sigma \\
\leq & \|V\|_{\infty} \int_{\Omega}(u)^{p(k+1)} d x+\left[|\lambda|\|m\|_{\infty}+|\mu|\right] \\
& \times \int_{\partial \Omega}\left(v_{M}\right)^{p(k+1)} d \sigma .
\end{aligned}
$$

Letting $M \rightarrow \infty$, using Fatou's Lemma we get

$$
\begin{aligned}
\frac{k p+1}{(k+1)^{p}} \int_{\Omega}\left|\nabla\left(u^{k+1}\right)\right|^{p} d x \leq & \|V\|_{\infty} \int_{\Omega}(u)^{p(k+1)} d x+\left[|\lambda|\|m\|_{\infty}+|\mu|\right] \\
& \times \int_{\partial \Omega} u^{p(k+1)} d \sigma .
\end{aligned}
$$

Set $A_{1}=\|V\|_{\infty} ; A_{2}=\left[|\lambda|\|m\|_{\infty}+|\mu|\right]$ and $A=\max \left\{A_{1}, A_{2}\right\}$. Then one has

$$
\frac{k p+1}{(k+1)^{p}} \int_{\Omega}\left|\nabla\left(u^{k+1}\right)\right|^{p} d x \leq A\left(\int_{\Omega} u^{p(k+1)} d x+\int_{\partial \Omega} u^{p(k+1)} d \sigma\right) .
$$

Since $\frac{k p+1}{(k+1)^{p}}<1$ for any $k>0$, adding $\int_{\Omega} u^{p(k+1)} d x$ to each side of above inequality to get

$$
\begin{aligned}
\frac{k p+1}{(k+1)^{p}}\left\|u^{k+1}\right\|_{W^{1, p}(\Omega)}^{p} & \leq(A+1) \int_{\Omega} u^{p(k+1)} d x+A \int_{\partial \Omega} u^{p(k+1)} d \sigma \\
& \leq(A+1)\left[\int_{\Omega} u^{p(k+1)} d x+\int_{\partial \Omega} u^{p(k+1)} d \sigma\right]
\end{aligned}
$$


Hence

$$
\left\|u^{k+1}\right\|_{W^{1, p}(\Omega)} \leq\left[\frac{(A+1)(k+1)^{p}}{k p+1}\right]^{\frac{1}{p}}\left[\int_{\Omega} u^{p(k+1)} d x+\int_{\partial \Omega} u^{p(k+1)} d \sigma\right]^{\frac{1}{p}} .
$$

By Sobolev's embedding theorem, there exists $C_{1}>0$ such that

$$
\left\|u^{k+1}\right\|_{L^{q}(\partial \Omega)} \leq C_{1}\left\|u^{k+1}\right\|_{W^{1, p}(\Omega)}
$$

which implies that

$$
\|u\|_{L^{q(k+1)}(\partial \Omega)}=\left\|u^{k+1}\right\|_{L^{q}(\partial \Omega)}^{\frac{1}{k+1}} \leq C_{1}^{\frac{1}{k+1}}\left\|u^{k+1}\right\|_{W^{1, p}(\Omega)}^{\frac{1}{k+1}} .
$$

Thus, we deduce from (6.1) that

$$
\begin{aligned}
& \|u\|_{L^{q(k+1)}(\partial \Omega)} \\
& \quad \leq C_{1}^{\frac{1}{k+1}}\left[\frac{(A+1)(k+1)^{p}}{k p+1}\right]^{\frac{1}{p(k+1)}}\left[\int_{\Omega} u^{p(k+1)} d x+\int_{\partial \Omega} u^{p(k+1)} d \sigma\right]^{\frac{1}{p(k+1)}} .
\end{aligned}
$$

On the other hand there exists $C_{2}>0$ such that

$$
\left[\frac{(A+1)(k+1)^{p}}{k p+1}\right]^{\frac{1}{p \sqrt{k+1}}} \leq C_{2} \text { for all } k>0 .
$$

and consequently

$$
\|u\|_{L^{q(k+1)}(\partial \Omega)} \leq C_{1}^{\frac{1}{k+1}} C_{2}^{\frac{1}{\sqrt{k+1}}}\left[\int_{\Omega} u^{p(k+1)} d x+\int_{\partial \Omega} u^{p(k+1)} d \sigma\right]^{\frac{1}{p(k+1)}}
$$

Choosing $k=k_{1}$ in (6.2) such that $\left(k_{1}+1\right) p=q$ then $(6.2)$ becomes

$$
\|u\|_{L^{q\left(k_{1}+1\right)}(\partial \Omega)} \leq C_{1}^{\frac{1}{k_{1}+1}} C_{2}^{\frac{1}{\sqrt{k_{1}+1}}}\left[\int_{\Omega} u^{q} d x+\int_{\partial \Omega} u^{q} d \sigma\right]^{\frac{1}{q}} .
$$

Next we choose $k=k_{2}$ in $(6.2)$ such that $\left(k_{2}+1\right) p=\left(k_{1}+1\right) q$ and get

$$
\begin{aligned}
\|u\|_{L^{q\left(k_{2}+1\right)}(\partial \Omega)} & \leq C_{1}^{\frac{1}{k_{2}+1}} C_{2}^{\frac{1}{\sqrt{k_{2}+1}}}\left[\|u\|_{L^{p\left(k_{2}+1\right)}(\Omega)}^{p\left(k_{2}+1\right)}+\|u\|_{L^{p\left(k_{2}+1\right)}(\partial \Omega)}^{p\left(k_{2}+1\right)}\right]^{\frac{1}{p\left(k_{2}+1\right)}} \\
& =C_{1}^{\frac{1}{k_{2}+1}} C_{2}^{\frac{1}{\sqrt{k_{2}+1}}}\left[\|u\|_{L^{\left(k_{1}+1\right) q(\Omega)}}^{\left(k_{1}+1\right) q}+\|u\|_{L^{\left(k_{1}+1\right) q}(\partial \Omega)}^{\left(k_{1}+1\right) q}\right]^{\frac{1}{\left(k_{1}+1\right) q}} .
\end{aligned}
$$

By induction we obtain

$$
\begin{aligned}
& \|u\|_{L^{q\left(k_{n}+1\right)}(\partial \Omega)} \\
& \quad \leq C_{1}^{\frac{1}{k_{n}+1}} C_{2}^{\frac{1}{\sqrt{k_{n}+1}}}\left[\|u\|_{L^{\left(k_{n-1}+1\right) q}(\Omega)}^{\left(k_{n-1}+1\right) q}+\|u\|_{L^{\left(k_{n-1}+1\right) q}(\partial \Omega)}^{\left(k_{n-1}+1\right) q}\right]^{\frac{1}{\left(k_{n-1}+1\right) q}}
\end{aligned}
$$

where $\left(k_{n}\right)$ is a sequence defined by

$$
k_{0}=0 \quad \text { and } \quad\left(1+k_{n}\right) p=\left(1+k_{n-1}\right) q \quad \forall n \geq 1 .
$$

One easily see that $1+k_{n}=\left(\frac{q}{p}\right)^{n} \quad \forall n \in \mathbb{N}$ and

$$
\|u\|_{L^{\alpha_{n}}(\Omega)} \leq C_{1}^{\beta_{n}^{2}} C_{2}^{\beta_{n}}\left[\|u\|_{L^{\alpha_{n-1}}(\Omega)}^{\alpha_{n-1}}+\|u\|_{L^{\alpha_{n-1}(\partial \Omega)}}^{\alpha_{n-1}}\right]^{\frac{1}{\alpha_{n-1}}}
$$


with $\beta_{n}=\frac{1}{\sqrt{1+k_{n}}}$ and $\alpha_{n}=\left(1+k_{n}\right) q$.

From the following embedding $W^{1, p}(\Omega) \hookrightarrow L^{r}(\Omega), \quad W^{1, p}(\Omega) \hookrightarrow L^{r}(\partial \Omega)$, for $p<r<p^{*}$ where $p^{*}=\frac{N p}{N-p}$, there exist $C_{3}, C_{4}>0$ such that

$$
\|u\|_{L^{\alpha}{ }^{\alpha-1}(\Omega)} \leq C_{3}\|u\| \quad \text { and } \quad\|u\|_{L^{\alpha_{n-1}(\partial \Omega)}} \leq C_{4}\|u\|
$$

Then

$$
\left[\|u\|_{L^{\alpha_{n-1}(\Omega)}}+\|u\|_{L^{\alpha_{n-1}(\partial \Omega)}}\right]^{\frac{1}{\alpha_{n-1}}} \leq 2^{\frac{1}{\alpha_{n-1}}} C_{5}\|u\|
$$

where $C_{5}=\max \left\{C_{3}, C_{4}\right\}$.

Relation (6.3) implies

$$
\|u\|_{L^{\alpha_{n}}(\partial \Omega)} \leq C_{1}^{\beta_{n}^{2}} C_{2}^{\beta_{n}} 2^{\frac{1}{\alpha_{n}-1}} C_{5}\|u\| .
$$

As $\frac{p}{q}<1$ then $\lim _{n \rightarrow+\infty} k_{n}=+\infty$ which implies that $\beta_{n} \rightarrow 0, \alpha_{n} \rightarrow+\infty$. So there exists $C>0$ such that

$$
\|u\|_{L^{\alpha_{n}(\partial \Omega)}} \leq C\|u\| \quad \text { for } \mathrm{n} \text { sufficiently large. }
$$

Suppose $u \notin L^{\infty}(\Omega)$ then there exists $\varepsilon>0$ and a set $\Theta$ of positive measure in $\Omega$ such that

$$
|u(x)| \geq C\|u\|+\varepsilon=K \quad \forall x \in \Theta
$$

Then

$$
\begin{aligned}
\liminf \|u\|_{L^{\alpha_{n}}(\partial \Omega)} & \geq \liminf \left(\int_{\Theta} K^{\alpha_{n}}\right)^{\frac{1}{\alpha_{n}}} \\
& =\liminf K|\Theta|^{\frac{1}{\alpha_{n}}} \\
& =K>C\|u\|,
\end{aligned}
$$

a contradiction with (6.5).

Having proved that any (weak) eigenfunction $u$ of $(1.1)$ is in $L^{\infty}(\Omega)$, we can use Theorem 2 in [19] to conclude that any weak solution of(1.1) $u$ is in $C^{1, \alpha}(\bar{\Omega})$ since $m \in C^{r}(\partial \Omega)$ (for some $r \in(0,1)$ ).

\section{References}

[1] Allegretto, W., Huang, Y.X.: A Picone's identity for the $p$-Lapalcian and applications. Nonlinear Anal. TMA 32(7), 819-830 (1998)

[2] Anane, A., Chakrone, O., Karim, B., Zerouali, A.: Eigencurves for Steklov problem. Electr. J. Diff. Equ. 75, 1-8 (2009)

[3] Arias, M., Campos, J., Cuesta, M., Gossez, J.-P.: Asymmetric elliptic problems with indefinite weights. Ann. Inst. H. Poincaré, An. Non Li. 19, 581-616 (2002)

[4] Arias, M., Campos, J., Cuesta, M., Gossez, J.-P.: An asymmetric Neumann problem with weights. Ann. Inst. H. Poincaré, An. Non Li. 25(2), 267-280 (2008) 
[5] Binding, P.A., Huang, Y.X.: Existence and nonexistence of positive eigenfunctions for the $p$-Laplacian. Proc. Am. Math. Soc. 123(6), 1833-1838 (1995)

[6] Binding, P.A., Huang, Y.X.: The eigencurve for the $p$-Laplacian. Diff. Int. Equ. 8(2), 405-414 (1995)

[7] Bonder, J.F., Rossi, J.D.: A nonlinear problem with indefinite weights related to the Sobolev trace embedding. Publ. Math. 46, 221-235 (2002)

[8] Cuesta, M., Ramos Quoirin, H.: A weighted eigenvalue problem for the p-Laplacian plus a potential. Nonlinear Diff. Equ. Appl. 16, 469-491 (2009)

[9] Dautray, R., Lions, J.L.: Analyse Mathématiques et calcul numérique pour les sciences et les techniques. Masson, Paris (1984)

[10] Dibenedetto, E.: $C^{1+\alpha}$ local regularity of weak solutions of degenerate elliptic equations. Nonlinear Anal. TMA 7, 827-850 (1983)

[11] El Khalil, A., Ouanan, M.: Boundary eigencurve problems involving the $p$-Laplacian operator. Electr. J. Diff. Equ. 78, 1-13 (2008)

[12] Garcia-Melian, J., Sabinade Lis, J.C.: Maximum and comparaison principles for operators involving the $p$-Laplacian. J. Math. Anal. Appl. 218, 49-65 (1998)

[13] Garcia-Melian, J., Rossi, J.D., Sabinade Lis, J.C.: A bifurcation problem governed by the boundary condition I. Nonlinear Diff. Equ. Appl. 14, 499-525 (2007)

[14] Godoy, T., Gossez, J.-P., Paczka, S.: A minimax formula for the principal eigenvalues of Dirichlet problems and its applications. 2006 International Conference in honor of Jacqueline Fleckinger. Electr. J. Diff. Equ. Conf. 16, 137-154 (2007)

[15] Hess, P., Kato, T.: On some linear and nonlinear eigenvalue problems with an indefinite weight function. Commun. Partial Diff. Equ. 5(10), 999-1030 (1980)

[16] Lindqvist, P.: On the equation $\operatorname{div}\left(|\nabla u|^{p-2} \nabla u\right)+\lambda|u|^{p-2} u=0$. Proc. Am. Math. Soc. 109, 157-164 (1992)

[17] Lindqvist, P.: Addendun in Proc. Am. Math. Soc. 116, 583-584 (1992)

[18] Lê, A.: Eigenvalue problems for the $p$-Laplacian. Nonlinear Anal. 64, 1057-1099 (2006)

[19] Liebarman, G.M.: Boundary regularity for solutions of degenerate elliptic equations. Nonlinear Anal. Theory Methods Appl. 12(11), 1203-1219 (1988)

[20] Leadi, L., Ramos quoirin, H.: Weighted asymmetric problems for an indefinite elliptic operator. Nonlinear Partial Differential Equations, Contemporary Mathematics, vol. 540, pp. 135-163. American Mathematics Society, Providence (2011)

[21] Lopez-Gomez, J.: The maximum principle and the existence of principal eigenvalues for some linear weighted boundary value problems. J. Diff. Equa. 127, 263294 (1995) 
[22] Serrin, J.: Local behavior of solutions of quasilinear equations. Acta Math. 111, 247-302 (1962)

[23] Steklov, M.W.: Sur les problèmes fondamentaux de la physique mathématique. Ann. Sci. Ecole Normale Sup. 19, 455-490 (1902)

[24] Struwe, M.: Variational methodsi In: Applications to Nonlinear PDE and Hamiltonial systems. Springer, Berlin (1983)

[25] Tolksdorf, P.: On the Dirichlet problem for quasilinear equations in domains with conical boundary points. Commun. PDE 8, 773-817 (1983)

[26] Tolksdorf, P.: Regularity for a more general class of quasilinear elliptic equations. J. Diff. Equ. 51, 126-150 (1984)

[27] Torné, O.: Steklov problem with an indefinite weight for the $p$-Laplacian. Electr. J. Diff. Equ. 87, 1-8 (2005)

[28] Vazquez, J.L.: A strong maximum principle for some quasilinear elliptic equations. Appl. Math. Optim. 12, 191-202 (1984)

[29] Ziemer, W.P.: Weakly differentiable functions. Graduate Texts in Mathematics, vol. 120. Springer, New York (1989)

Liamidi Leadi and Aboubacar Marcos

Institut de Mathématiques et de Sciences Physiques

Université d'Abomey-Calavi

01 BP 613 Porto-Novo

Republic of Benin

e-mail: leadiare@imsp-uac.org;

abmarcos@imsp-uac.org

Received: 26 July 2011.

Accepted: 24 May 2012. 